\title{
Rice LEAFY COTYLEDONE1 regulates embryonic envelope development and chlorophyll biogenesis in embryo
}

Chen Chen ( $\square$ chenchen@yzu.edu.cn )

Yangzhou University https://orcid.org/0000-0002-9748-3111

Baixiao Niu

Yangzhou University

Zongju Yang

Yangzhou University

Tianqi Bai

Yangzhou University

Zhiguo E

China National Rice Research Institute

Xinyu Xu

Yangzhou University

Qianbin Yun

Yangzhou University

Juan Zhang

Yangzhou University

Zhenyu Zhang

Yangzhou University

Xinming Lu

Yangzhou University

Qianfeng Li

Yangzhou University

Qiaoquan Liu

Yangzhou University https://orcid.org/0000-0001-5543-5798

\section{Article}

Keywords: chlorophyll biogenesis, plant genetics, embryonic envelope

Posted Date: September 22nd, 2021

DOl: https://doi.org/10.21203/rs.3.rs-888827/v1 
License: (c) (i) This work is licensed under a Creative Commons Attribution 4.0 International License. Read Full License 
1 Rice LEAFY COTYLEDONE1 regulates embryonic envelope development and chlorophyll

2 biogenesis in embryo

3 Baixiao $\mathrm{Niu}^{1,{ }^{+}}$, Zongju Yang ${ }^{1{ }^{+}}$, Tianqi $\mathrm{Bai}^{1{ }^{1,}}$, Zhiguo $\mathrm{E}^{2}$, Xinyu $\mathrm{Xu}^{1}$, Qianbin Yun ${ }^{1}$, Juan $\mathrm{Zhang}^{1}$,

4 Zhenyu Zhang ${ }^{1}$, Xinming $\mathrm{Lu}^{1}$, Qianfeng $\mathrm{Li}^{1}$, Qiao-Quan $\mathrm{Liu}^{1}$, Chen Chen ${ }^{1, *}$

$5{ }^{1}$ Jiangsu Key Laboratory of Crop Genetics and Physiology/ Key Laboratory of Plant Functional

6 Genomics of the Ministry of Education/ Jiangsu Key Laboratory of Crop Genomics and Molecular

7 Breeding/ Jiangsu Co-Innovation Center for Modern Production Technology of Grain Crops,

8 Agricultural College of Yangzhou University, Yangzhou, China

$9 \quad{ }^{2}$ Key Laboratory of Rice Biology, China National Rice Research Institute, Hangzhou, China

${ }^{\dagger}$ These authors contributed equally to this work

* Correspondence: chenchen@yzu.edu.cn 
The scutellum, coleoptile, coleorhiza, and epiblast (if it exists) consist of a complex embryonic envelope to protect the plumule and radicle inside a grass embryo. Controversies have been provoked for centuries regarding homologies of the grass embryonic structures. Here we found that the rice LEAFY COTYLEDONE1 (LEC1) gene, OSNF-YB7, is vital for embryo development. A leaf-like structure (LL) was developed from the scutellum of $o s n f-y b 7$ to replace the embryonic envelope that formed in wild-type. Additionally, osnf-yb7 developed chloroembryos due to overactivated chlorophyll biosynthesis. Thus, OsNF-YB7 likely plays a dual role in chlorophyll biogenesis in rice embryos: (1) OsNF-YB7 directly represses genes, such as rice GOLDED-LIKE1 (OsGLK1), involving chlorophyll biosynthesis; (2) OsNF-YB7 binds to OsGLK1 to repress the downstream genes of OsGLK1. Parallel phenotypes shown in osnf-yb7 and lec1 suggest functional conservation of the LEC1-type genes in plants. Both lec1 cotyledons and osnf-yb7 LL displayed true leaf characteristics. Our morphological and transcriptional evidence implied that LL replaces the embryonic envelope in osnf-yb7, raising the hypothesis that the grass embryonic envelope is an analog of Arabidopsis cotyledon. This study demonstrates that OsNF-YB7 acts as a negative regulator in chlorophyll biogenesis and is important for embryonic envelope formation. 


\section{Introduction}

LEAFY COTYLEDON1 (LEC1) of Arabidopsis encodes a subunit of the nuclear family $\mathrm{Y}$ (NF-Y) transcription factor (TF) with specialized function in plants (Lotan et al., 1998; Lee et al., 2003). LEC1 and the B3 family TFs, including LEC2, ABA INSENSITIVE3 (ABI3), and FUSCA3 (FUS3), consist of a central hub for regulating the network of seed maturation (Jo et al., 2019; He and Niu, 2019; Lepiniec et al., 2018). Loss-of-function of LEC1 results in desiccation intolerance, defects of storage macromolecule accumulation, activation of the shoot apices, and precocious germination (Meinke, 1992; Meinke et al., 1994; West et al., 1994; Mu et al., 2008; Harada, 2001). LEC1 is also involved in Arabidopsis embryogenesis (Hu et al., 2018). The suspensor of the lec1 embryo had more cells due to abnormal cell division (Meinke, 1992; Meinke et al., 1994; West et al., 1994); trichomes, the unicellular outgrowth from the epidermis of true leaves, were ectopically developed on the surface of the lec1 cotyledons (Meinke et al., 1994; West et al., 1994), indicating that heterochronic conversion was induced. Recent studies have revealed that LEC1 transcriptionally activates chloroplast biogenesis and photosynthesis in Arabidopsis (Pelletier et al., 2017; Jo et al., 2020), consistent with the observation that the lec1 showed paler green coloration than wild-type (WT) embryos (Meinke, 1992; West et al., 1994). Intriguingly, LEC1 can also act as a pioneer TF to regulate flowering by reprogramming embryonic chromatin states (Tao et al., 2017).

Grass embryos are distinct from those of dicots and other monocots in many aspects (Armenta-Medina et al., 2021); for example, the grass embryo has a more complex structure. An embryonic envelope, consisting of the scutellum, coleoptile, coleorhiza, and epiblast (if it exists in the embryo of the species), is formed in grass to protect the plumule and radicle inside (Xu et al., 1999). Additionally, grass embryos can develop foliage leaves coupled with embryogenesis, indicating that early vegetative development is incorporated into the embryo development (Itoh et al., 2005). Moreover, cell division during Arabidopsis embryogenesis is stereotypical; however, for grass, such as rice and maize, there is no set pattern for the zygotic division after the first asymmetry division (Ishimoto et al., 2019).

According to morphological characteristics and landmark events, rice embryo development can 
be divided into ten stages (Itoh et al., 2005). After fertilization (stage Em1), a globular embryo is developed around three days after fertilization (DAF) (Em2-Em4). The onset of coleoptile and the differentiation of the shoot apical meristem (SAM) and radical are then observed at 4 DAF (Em5). At 5-6 DAF, the first leaf is formed (Em6), and at 7-8 DAF, three foliage leaves are recognizable in the shoot apex (Em7). After that, there are no more morphological changes other than the remarkably enlarged embryo (Em8). Finally, the embryo gradually matures from 11 to 20 DAF (Em9) and acquires dormancy after then (Em10).

Hundreds of mutants showing abnormal embryo development have been isolated in rice (Hong et al., 1995; Nagato et al., 1989). In the past two decades, several key genes governing rice embryogenesis, embryo patterning, and organogenesis have been cloned, substantially expanding our understanding of the embryo development regulation in rice at a molecular level (Ishimoto et al., 2019; Yi et al., 2016; Sazuka et al., 2009; Nosaka et al., 2007; Hibara et al., 2009; Qi et al., 2020; Huang et al., 2017). However, ironically, several fundamental questions, such as identities and their homologies of the scutellum, coleoptile, coleorhiza, and epiblast, remained unanswered, although the debate has been provoked for centuries (Brown, 1960, 1965; Xu et al., 1999). Some mutants showing embryogenesis defects may provide novel perspectives for such questions. For example, the rice shootless (shl) mutants can develop scutellum but cannot form epiblast and coleoptile in the embryo, indicating that differentiation of the epiblast and coleoptile depends on the SAM, but that of the scutellum is regulated independently of SAM (Satoh et al., 1999). However, weak alleles of the shl mutants could form the SAM, but no coleoptile was produced (Satoh et al., 2003), suggesting that the coleoptile likely has a different identity to that of SAM-derived leaves. The unavailability of more informative mutants, such as shl, limits further discussions.

The angiosperm can be divided into chloroembryophytes and leucoembryophytes, depending on the presence and absence of chlorophyll (Chl) in embryos, respectively (Smolikova and Medvedev, 2016; Puthur et al., 2013). Some plant species, such as Arabidopsis, produce Chl when the embryo develops. As in leaves, Chl synthesized in the embryo also shows photosynthesis ability (Simkin et al., 2020). However, grass species, such as rice, cannot produce Chl when their embryos develop. To the best of our knowledge, what determines Chl 
biosynthesis ability in plant embryos is completely unknown; however, several genes that contribute to Chl degradation for chloroembryos, whose mutation can lead to a stay-green phenotype of mature seeds, have been identified (Smolikova et al., 2017).

There are two LEC1-type genes, OsNF-YB7 and OsNF-YB9, encoded by the rice genome (E et al., 2018). We previously found that OsNF-YBT or OsNF-YB9 could recover the LEC1 defects of the Arabidopsis mutant, lec1-1 (Niu et al., 2021), indicating that the function of LEC1-type genes in rice and Arabidopsis is conserved. The LEC1-type genes, in Arabidopsis or rice, display a seed-preferential expression pattern. However, OsNF-YB7 is predominately expressed in the embryo, while OsNF-YB9 shows an endosperm-preferential expression (Niu et al., 2021). Given their distinct expression patterns in seeds, we assumed that OsNF-YB7 and OsNF-YB9 had been subfunctionalized during evolution. Null mutations of OsNF-YB9 resulted in reduced seed size and increased chalkiness of rice seeds but showed no effects on seed viability (Niu et al., 2021). However, osnf-yb7 loss-of-function seeds cannot germinate using undetected mechanisms (Niu et al., 2021). This study found that osnf-yb7 showed phenotypes similar to that of Arabidopsis lec1, such as dessication intolerance, reduced dormancy, and embryogenesis defects. Intriguingly, the coleoptile, epiblast, and coleorhiza were not observable in an osnf-yb7 embryo, instead, a true leaf-like structure (LL) was developed from the scutellum. This allows us to discuss the homologies of complex embryo structures in grass; additionally, we found that osnf-yb7 produced chloroembryos. As far as we know, this phenotype has never been reported in grass. Our findings suggested that OsNF-YB7 can repress the OsGLK1-mediated chlorophyll biosynthesis pathway in embryos, directly and indirectly.

\section{Results}

\section{OsNF-YBT is required for embryonic envelope development in rice}

Previous studies have suggested that either knockout or knockdown of OsNF-YB7 in rice is lethal (Niu et al., 2021; Ito et al., 2011; Zhang and Xue, 2013). To explore the cause of lethality, we analyzed embryo development in the osnf-yb7 mutants that we previously generated (Niu et al., 2021). By confocal laser scanning microscopy (CLSM) observations, we found that there was no developmental difference between the mutant and WT embryos before 4 DAF (Fig. 1A-F). In the 
5-DAF-old WT embryos, the coleoptile protruded from the ventral region above the SAM, which, nevertheless, was not observed in the mutant (Figs. 1G and H). Because osnf-yb7-1 and osnf-yb7-2, two independent mull mutant lines, showed consistent phenotypes (Fig. S1A-F), we mainly used osnf-yb7-1 for subsequent analyses. From a ventral side view of scanning electron microscopy (SEM), we found that the dome-like coleoptile of WT was surrounded by the scutellum at 5 DAF and protected by the ventral and lateral scales derived from the scutellum at 10 DAF (Fig. 1I-L). However, osnf-yb7 embryos developed a flat leaf-like structure (LL) from the scutellum enclosing the shoot apex of mutant embryos (Fig. 1M-P). Investigation of the vertical and transverse sections further confirmed that the coleoptile was not developed, but replaced with a LL, in the osnf-yb7 embryos (Fig. 1Q-T). Notably, the onset of LL in osnf-yb7 coincided with coleoptile initiation in WT (Figs. 1R and T), indicating that LL is an analog or a heteromeric organ of the coleoptile. To unravel the LL's identity, we morphologically and histologically characterized it after germination (Fig. 2A-L). Hairs developed at the apical tip of LL, and trichomes were found on the LL's surface (Figs. 2D, H and I). The hair and trichomes do not develop on the WT coleoptile but can be found on the first leaf (Fig. 2E-H). These findings suggested that the $\mathrm{LL}$ resembles a leaf rather than a coleoptile. Additionally, the cell shape, cell composition, and cell arrangement of the LL were more similar to that of the first leaf of WT (Figs. $\mathbf{2 H}$ and $\mathrm{J})$. Moreover, we found that there were multiple vascular bundles formed in the $\mathrm{LL}$ of osnf-yb7, whereas there were only two developed in a WT coleoptile (Fig.2K and L).

No epiblast and coleorhiza were formed in the embryos produced by osnf-yb7 (Fig. 11-T). The hypocotyl of osnf-yb7 was strikingly longer than that of WT (Figs. 1Q and S). As indicated by the $\mathrm{I}_{2}-\mathrm{IK}$ and coomassie brilliant blue staining assays, the scutellum of osnf-yb7 accumulated significantly less starch grains and protein in the parenchyma cells (Fig. S2A-D). The WT scutellum differentiated a layer of palisade-shaped epithelial cells that could be observed in an 8-DAF-old embryo; however, the mutant scutellar epithelium was not strikingly different from the inner parenchyma cells regarding cell shape (Fig. S2B and D). It is worth noting that SAM establishment was not impaired in osnf-yb7, given that the onset of leaf primordium was normal in 5-DAF-old embryos (Figs. S2E and F). These findings suggested that OsNF-YB7 is indispensable for embryogenesis in rice. 
151

We next performed RNA-seq analysis to investigate the transcriptomic changes in 5- and 10-DAF-old embryos of osnf-yb7. There were 1,311 and 3,006 genes down- and upregulated in the 5-DAF-old embryos of osnf-yb7, respectively, compared with the WT embryos at the same age, using the threshold fold change $>2$ and Bonferroni $p<0.05$ (Table S1). Similarly, 1,280 and 4,100 genes were down- and upregulated, respectively, in the osnf-yb7 embryos at 10 DAF (Table S2). However, most of the differentially expressed genes (DEGs) identified at 5 and 10 DAF were common (Fig. 3A). Notably, the number of upregulated genes was consistently higher than that of downregulated ones, indicating that OsNF-YB7 mainly functions as a transcriptional repressor in rice embryos. Interestingly, we found that the most upregulated genes at 10 DAF, whose fold changes ranked the top 100 in osnf-yb7, are usually highly expressed in vegetative tissues but showed less expression in the embryo (Fig. 3B). Instead, the most downregulated genes (top 100) display an overall embryo or caryopsis preferential pattern (Fig. 3B). This provides transcriptional evidence for the assumption that the LL of osnf-yb7 is more like a vegetative (i.e., leaf) than an embryonic structure.

Itoh et al. (2016) identified a set of genes showing tissue-specific upregulation in the shoot, scutellum, root, or epiblast/coleorhiza in the embryo. We found that approximately $40 \%$ of these genes displayed differential expression in the osnf-yb7 embryonic tissues (Table S3), suggesting that the OSNF-YBT defects resulted in extensive deregulation of embryogenesis. In a genome-wide analysis, approximately $30 \%$ and $23 \%$ of the DEGs we identified in 5 - and 10-DAF-old mutant embryos, respectively, were downregulated (Fig. 3A). However, for the scutellum, preferential genes more than $52 \%$ and $42 \%$ DEGs were downregulated at 5 and 10 DAF (Figs. 3C and D), respectively, indicating that the downregulated genes were over-represented in the scutellum of osnf-yb7. In contrast, only a small portion of the epiblast (9\%-16\%) and root preferential DEGs (13\%-16\%) were downregulated (Fig. 3C and D). The results implied that the scutellum showed unique expressional changes in osnf-yb7, in line with OsNF-YB7 is a scutellum-preferential gene (Niu et al., 2021; Zhang and Xue, 2013). 
PlantTFDB, 359 and 389 showed differential expression in 5- and 10-DAF-old osnf-yb7 embryos, respectively, compared with the WT (Table S4). Distinct TF families were enriched in the upregulated and downregulated TFs. For example, AP2, B3, NF-YA, ARF, and GRF family TFs were over-represented in the downregulated DEGs; whereas, AUX/IAA and RAV TF family members were over-represented in the upregulated DEGs that were identified from the osnf-yb7-1 embryos (Fig. 3E and F). Notably, many differentially expressed TFs encode homeobox members that play essential roles in embryogenesis and embryo patterning (Table S4). For example, the ZF-HD subfamily genes were enriched in the downregulated DEGs at 5 and 10 DAF (Fig. 3E and F). A group of WOX genes was also suppressed in the mutant, although only enriched at 5 DAF (Fig. 3E). In contrast, HD-ZP subfamily TFs were over-represented in the upregulated DEGs at 5 and 10 DAF (Fig. 3E and F). Likewise, many three-amino-acid-loop-extension classes of homeobox genes, including the KNOTTED-like (KNOX) homeodomain and BEL1-like (BELL) homeodomain members, were activated in the 10-DAF-old osnf-yb7-1 embryo (Fig. 3F).

\section{The embryo of osnf-yb7 remains active after embryogenesis}

For WT, there were 3,266 and 1,193 genes down- and upregulated in the embryos at 10 DAF compared with the mRNA profiles of 5-DAF-old embryos (Fig. 3A, Table S5). However, for osnf-yb7, the number of down- and upregulated genes identified between 5- and 10-DAF-old mutant embryos were 1,553 and 999, respectively (Fig. 3A, Table S6). The results suggested that more genes were transcriptionally repressed at 10 DAF in WT than in osnf-yb7. Because these DEGs reflected transcriptional changes in different developmental stages (10 DAF vs. 5 DAF), we termed them development-related DEGs (drDEGs). Intriguingly, Gene Ontology (GO) analyses showed that distinct biological processes were enriched for the WT and osnf-yb7 drDEGs (Fig. 3G). For example, the terms "response to stimulus" and "stress response" were enriched for the upregulated drDEGs in WT, which is expected due to dehydration onset in embryos at 10 DAF. However, the same terms were enriched for the downregulated-, but not for the upregulated drDEGs, identified in osnf-yb7 (Fig. 3G). Genes involving expression and translation were over-represented for the upregulated drDEGs in osnf-yb7, and the downregulated drDEGs in WT

(Fig. 3G). Moreover, several terms associated with active cell activities, such as "signal transduction" and "cell cycle," were specifically enriched for the upregulated drDEGs in osnf-yb7 
(Fig. 3G). The transcriptional differences suggested that osnf-yb7 embryos were more active than WT at 10 DAF. Consistent with this, a 2,3,5-triphenyltetrazolium chloride (TTC) staining assay showed that staining reactions in the shoot and root of the WT 10-DAF-old embryos were not as active as that reacted in the mutant, indicating the osnf-yb7 embryos were metabolically overactivated (Fig. 3H and I). After finishing embryogenesis, there was no morphological change in a WT rice embryo (Itoh et al., 2005). However, because the developmental cessation was largely lost in osnf-yb7, many morphological variations could be observed for the mutant embryos at $\square 15$ DAF (Fig. 3J and K).

\section{Loss-of-function of OSNF-YB7 weakens dormancy}

Persistent growth of the osnf-yb7 embryos indicates that dormancy was a breakdown in the mutants. Consistent with this, several key genes involving rice dormancy, such as OsVP1/OsABI3 (Wang et al., 2020; Sugimoto et al., 2010; Chen et al., 2021), OsFLF1, and OsGD1 (Guo et al., 2013), are significantly suppressed in the mutant (Fig. S3A-C). However, we found that many genes governing ABA biosynthesis were overall activated in the mutant (Fig. 4A and B), which is possibly feedback to the decreased ABA content in osnf-yb7 (Fig. 4C). Furthermore, some ABA-responsive genes, including OsVP1/OsABI3, OsABI4/OsERF117, and OsABI5/OsZIP10, and their downstream genes, such as Sdr4, were significantly suppressed in the osnf-yb7 embryos, at either 5 or 10 DAF (Fig. 4 B). However, we found that some key components involving stress-induced ABA signaling were activated, rather than inactivated, in osnf-yb7. For example, OSBZ8/OsbZIP05, OsABIL1,2,3, OsABF1/OsbZIP12, and OsABF2/OsbZIP46 showed significantly higher expression in the mutant at 10 DAF (Fig. S3D-F, Table S7). These findings strongly indicated that OsNF-YB7 selectively repressed dormancy-related ABA signaling genes, such as OsVP1/OsABI3, OsABI4/OsERF117, and OsABI5/OsZIP10, in the embryo.

GA acts as an intrinsic signal to break dormancy; OsGA20ox1 and OsGA3ox2, two important GA biosynthetic genes were significantly upregulated in osnf-yb7 (Fig. 4B, Figs. S4A and B, and Table S8). In line with this, $\mathrm{GA}_{1}$ and $\mathrm{GA}_{4}$, two active $\mathrm{GA}$ molecules in rice, were highly accumulated in the osnf-yb7 embryo at 10 DAF (Fig. 4D and E). The ratio, ABA:GA, which is important for maintaining seed dormancy, was substantially decreased in the mutant (Fig. 4F) due to the 
decreased $A B A$ and increased GA content in the mutant. Previous studies showed that the germination of seeds is associated with the activation of RAmy1A, an $\alpha$-amylase glycoprotein induced by the GA-induced GAMYB in rice (Morita et al., 1998). GAMYB was significantly activated in the mutant embryos, and accordingly, RAmy1A was highly expressed in osnf-yb7-1, whereas there was no RAmy1A transcript detectable in the WT embryo at 10 DAF (Fig. S4C and Table S8). Altogether, we assumed that the breakdown of osnf-yb7 dormancy was caused by activating GA biosynthesis, coupled with the repression of the ABA-induced dormancy signals.

\section{OsNF-YB7 is essential for the acquisition of desiccation tolerance}

Although osnf-yb7 embryo is more active after embryogenesis (Fig. 3G-K), the TTC staining analysis showed that mature seeds of osnf-yb7 were less vigorous than the WT after imbibition for $12 \mathrm{~h}$ (Fig. 4G and $\mathbf{H}$ ). Therefore, most of the dehydrated osnf-yb7 seeds are not germinable (Niu et al., 2021). However, we found that if the seeds were not desiccated, osnf-yb7 could germinate. Furthermore, using the seeds harvested before drying ( $\square 25$ DAF), osnf-yb7 germinated much faster than WT seeds (Fig. 4I). However, the subsequent seedling development of osnf-yb7 was arrested (Fig. 4J).

The findings suggested that $O S N F-Y B 7$ is required for the acquisition of desiccation tolerance in rice. In favor of this, the genes encoding late embryogenesis abundant (LEA) proteins that accumulate during late stages of embryogenesis and associate with dehydration were significantly repressed in the 10-DAF-old mutant embryo. However, there was no expression difference of these genes between the WT and osnf-yb7 embryos at 5 DAF (Fig. S5A). Among these LEA-encoding genes, OSEM, Rab16A, and REG2 are marker genes activated at the Em8 phase (Itoh et al., 2005). However, their expressions were largely repressed in osnf-yb7 embryos at 10 DAF (Fig. S5B-D).

\section{OsNF-YB7 represses chlorophyll biogenesis and photosynthesis in the embryo}

The embryos produced by osnf-yb7 embryos were greenish owing to the activation of $\mathrm{Chl}$ biogenesis (Fig. 5A-D). Intriguingly, the quantification analysis showed that the content of Chlb was not strikingly different between osnf-b7 and WT, but the mutant embryos accumulated 
more Chla, at either $\square 15$ DAF or maturation stage (Fig. 5C and D). Furthermore, chlorophyll autofluorescence could be observed in an osnf-yb7 embryo at as early as 5 DAF (Fig. S6A and B). Additionally, many genes responsible for rice chlorophyll biogenesis were activated in the mutant embryos at either 5- or 10 DAF (Fig. S6E and F). In consequence, as revealed by the Mapman analysis, genes involving photosynthesis were dramatically enriched for DEGs, and the vast majority of the genes was upregulated (Fig. S7A-D).

Several TFs have been identified in plants for regulating chlorophyll biogenesis or chloroplast development (Jarvis and López-Juez, 2013). Some TFs, such as rice GOLDEN 2-like1 (OsGLK1), HY5-like1 (OsHY5L1), PIF-like14 (OSPIL14), and rice GATA Nitrate-inducible Carbon-metabolism-involved (OsGNC), were significantly upregulated in the osnf-yb7 embryos; among which, OsGLK1 was the most activated gene (Fig. $5 \mathrm{E}-\mathbf{H}$ ). Overexpression of OsGLK1 can significantly increase chlorophyll content in rice (Nakamura et al., 2009). By surveying the genes upregulated in an OsGLK1 overexpression line (Nakamura et al., 2009), we found that most of the OsGLK1 activated genes ( $\square 70.2 \%$ ) were also upregulated in the osnf-yb7 embryos at either 5or 10-DAF (Fig. 5I). To explore the possibility that OsNF-YB7 directly represses the expression of OsGLK1, we conducted a dual-luciferase (LUC) assay to test the hypothesis. The results showed that OsNF-YB7 significantly repressed the activities of the LUC reporter driven by the OsGLK1 promoter (Fig. 5J and K).

Meanwhile, we examined the interaction between OsNF-YB7 and OsGLK using yeast two-hybrid assays $(\mathrm{Y} 2 \mathrm{H})$. We fused full-length OsGLK1 with the GAL4 DNA-activation domain and full-length OsNF-YB7 with the GAL4 DNA-binding domain. The results showed that OsNF-YB7 interacts with OsGLK1 in yeast (Fig. 6A). The proteins also showed strong interactions in the epidermal cells of tobacco, as indicated by the split complementary LUC assay (Fig. 6B). The bimolecular fluorescent complementary (BiFC) analysis suggested that the interaction occurred exclusively in the nuclei (Fig. 6C). Moreover, we transiently coexpressed OsNF-YB7 that tagged with green fluorescent protein (YB7-GFP), and OsGLK1 that tagged with 3x flag (GLK1-flag) in rice protoplast. Also, the co-immunoprecipitation (CoIP) analysis showed that GLK1-flag could be co-immunoprecipitated in rice using the anti-GFP antibody (Fig. 6D). Collectively, these findings suggest that OsNF-YB7 interacts with OsGLK1 both in vivo and in vitro. 
The primary target genes of GLK1 in Arabidopsis are light-harvesting and Chl biosynthesis-related genes (Nagatoshi et al., 2016). For example, the genes encoding protochlorophyllide oxidoreductase (POR), which catalyzes the reactions of protochlorophyllide (Pchlide) to chlorophyllide for Chl synthesis, were transcriptionally activated by GLK1 in Arabidopsis (Waters et al., 2009). Therefore, OsPORA and LHCB4 (Light-Harvesting Complex B4), a Chla-b binding protein-encoding gene that activated by OsGLK1 in rice (Zhang et al., 2021), were selected to analyze whether OsNF-YB7 disturbs the OsGLK1-mediated chlorophyll biosynthesis, given their expression was substantially activated in the osnf-yb7 embryos (Fig. 6E and F). As expected, the transient expression of OSGLK1 in rice protoplast substantially activated the reporter gene driven by the promoters of OSPORA and $\angle H C B 4$, indicating that these genes are transcriptionally targeted by OsGLK1 (Fig. 6H-I). When we coexpressed OsNF-YB7 with OsGLK1, the activation ability of OsGLK1 was significantly repressed (Fig. 6H-I). However, OsNF-YB7 alone did not show influences on the expression of the reporter (Fig. $6 \mathbf{H}-\mathbf{I}$ ). Altogether, we assumed that OsNF-YB7/OsGLK1 dimerization reduces the ability of OsGLK1 for Chl biogenesis.

Collectively, our results suggest that the activation of OSGLK1, at either transcriptional or post-translational level, contributes to $\mathrm{Chl}$ accumulation in the osnf-yb7 embryos. To test this, we generated high-order mutants of OsNF-YB7, OsGLK1, and OsGLK2, considering there are redundancies between OsGLK1 and OsGLK2 (Sakuraba et al., 2017; Wang et al., 2013). Using the CRISPR/Cas9 cassette that included three tandemly arrayed guide RNAs targeting OsNF-YB7, OsGLK1, and OsGLK2, respectively, we successfully obtained the osnf-yb7;osglk2 double mutant and the osnf-yb7;osglk1;osglk2 triple mutant at the $\mathrm{T}_{0}$ generation (Fig. S8). By analyzing embryos the mutants produced, we found that osnf-yb7;osglk2 and osnf-yb7;osglk1;osglk2 displayed embryo morphology similar to that of osnf-yb7 (Fig. 6J-M). However, the Chl accumulated in osnf-yb7;osglk1;osglk2 was less than in osnf-yb7 and osnf-yb7;osglk2 (Fig. 6J-M). In comparison to the achlorophyllous embryo of WT, the osnf-yb7;osglk1;osglk2 triple mutant still showed somewhat green coloration in apical part of the embryos (Fig. 6M), suggesting that in addition to OsGLKs, there are some other players contribute to the $\mathrm{Chl}$ biogenesis in the embryos of osnf-yb7. 

Zm00001d051697 and Zm00001d017898 are phylogenetically closer to OsNF-YB7, while Zm00001d045772 is a homolog of rice OsNF-YB9 (Fig. 7A). Similar to their rice homologs, Zm00001d051697 and Zm00001d017898 are predominantly expressed in the embryo, whereas Zm00001d045772 is endosperm preferentially expressed in our previous report (E et al., 2018). To detect whether Zm00001d051697 and Zm00001d017898 play a conserved role in embryo development, we generated double mutants of the genes using a CRIPSR/Cas9 gene-editing approach. Because of high similarities between Zm00001d051697 and Zm00001d017898, we could use the same guard RNA to knock out the two genes simultaneously (Fig. S9A). To increase the chance to obtain double mutants, we designed two targets for gene editing. We obtained five independent transgenic events, two of which produced seeds showing similar phenotypes to that of the osnf-yb7 mutants. By sequencing the $T_{2}$ individuals showing a phenotype, we found that both Zm00001d051697 and Zm00001d017898 were mutated, while the ones with only one gene-edited showed normal development (Fig. S9B).

All seeds produced by line A05-01-1 exhibited embryo defects. We, therefore, used this line for subsequent phenotypic analyses. Like that of osnf-yb7, post-germination development of A05-1-1 was arrested (Fig. 7B). No coleoptile was developed in the mature maize mutant seeds; instead, we could observe a LL structure after imbibition (Fig. 7C-H). The maize embryo does not have an epiblast, but we could still find that the structure surrounding the plumule-radicle axis at the ventral side was not developed in the lec1 mutant (Fig. $\mathbf{7 C}, \mathbf{D}, \mathbf{G}$, and $\mathbf{H}$ ), resembling the observation that epiblast was disappeared in the osnf-yb7 embryos. Similarly, the coleorhiza of the lec1 mutant in maize was also mal-developed (Fig. 7C and D). However, some of the dried mutant seeds were germinable and can develop into a fertile plant (Fig. S9C). A close observation of the germinated lec1 maize seeds suggested that a LL structure replaced the coleoptile, leaving the inside leaves unprotected (Fig. 7I-L). The cross-sections of germinated maize embryos showed that the LL structure of the lec1 mutant is a scutellum derivative (Fig. 7K and L), consistent with the phenotype that showed in the rice mutant. Notably, the maize lec1 embryos were achlorophyllous (Fig. 7C-H). This was possible because multiple layers of bracts inhibited light from penetrating the ear. In favor of this hypothesis, blocking light perception by covering 
aluminum foils on a rice panicle resulted in achlorophyllous embryos of osnf-yb7 (Fig. S10). Altogether, phenotypical similarities between the rice and maize lec1 mutants strongly suggested that the LEC1-type genes are functionally conserved for grass embryo development.

\section{Discussion}

\section{LEC1-type genes play a conserved role in plant seed development}

LEC1-type genes originated from non-LEC1 type NF-YB genes in basal land plants (Xie et al., 2008; Cagliari et al., 2014) and possibly played a crucial role in the evolution of seed in plants (Jo et al., 2019). The LEC1-type genes in grass species have been divergent into two subgroups: OsNF-YB7-like and OsNF-YB9-like (E et al., 2018). OsNF-YB7-like genes are phylogenetically closer to LEC1 and L1L of Arabidopsis, whereas the OsNF-YB9 homologs are found only in grass, indicating that OsNF-YB9 was evolved after the divergence of grass. LEC1 is expressed in the embryo and endosperm soon after fertilization in Arabidopsis (Xie et al., 2021). OsNF-YB7 exclusively expresses in the embryo, while the OsNF-YB9 are endosperm preferentially expressed in rice (Niu et al., 2021). Differentiated expression patterns of the OsNF-YB7-like and OsNF-YB9-like genes were also found in maize, sorghum, and barley (E et al., 2018). These different expression patterns suggest subfunctionalization of the LEC1-type genes in grass. A recent study showed that the endosperm-expressed $L E C 1$ in Arabidopsis is sufficient for embryo maturation (Xie et al., 2021). In the case of rice, osnf-yb9 showed no embryo defects (Niu et al., 2021); as well, osnf-yb7 showed no endosperm phenotype (Fig. S1A and Fig. S11A). Moreover, by investigating the expression profiles in the osnf-yb7 endosperm at $10 \mathrm{DAF}$, we found that only a small number of genes showed differential expression (88 downregulated and 136 upregulated) in comparison to the WT, further confirming that OsNF-YBT and OsNF-YBS are confined to different compartments (i.e., embryo and endosperm, respectively) for function (Fig. S11B and Table S9). However, we did find expression changes of some genes. For example, OsNF-YB9 was significantly activated in the osnf-yb7 endosperm, while several genes in response to auxin were downregulated (Fig. S11C).

We previously reported that OSNF-YBT and OSNF-YB9 could complement the lec1 defects in Arabidopsis (Niu et al., 2021). Here we found that almost all developmental defects showed in 
lec1 could be observed in osnf-yb7, including loss of quiescence, weakened dormancy, and desiccation intolerance (Figs. 3 and 4). Embryogenesis defects of lec1 and osnf-yb7 were also comparable. Both LEC1-type mutants showed over-proliferation of suspensors; the hypocotyl of the mutants was not as bent as the WT (Fig. 1I-T). A heterochronic conversion was induced in lec1, for whose cotyledons acquired characteristics displayed in a true leaf (Meinke et al., 1994; West et al., 1994). Likewise, an LL was derived from the osnf-yb7 scutellum at the corresponding regions where WT's coleoptile and epiblast originated. Therefore, we believed that the LL of osnf-yb7 is not a coleoptile analog, but more like a true leaf in several aspects (Fig. 2A-L): (1) trichomes were developed at the tip and on the surface of the LL structure; (2) cell arrangement of the LL structure was different from that of the coleoptile; (3) multiple vascular bundles were developed rather than two. This evidence suggested a leaf identity of LL. Additionally, the maize lec1 mutants, we generated also displayed similar embryo morphology: the coleoptile was substituted by a structure similar to foliage (Fig. 7B-L).

\section{Reconsideration of the homologies of complex embryo structures in grass}

Several distinct concepts have been proposed to interpret what the grass embryonic structures represent. The scutellum is considered equivalent to the cotyledon, either entire or partial of it (Brown, 1965, 1960; Xu et al., 1999; Chandler, 2008; Satoh et al., 1999). However, no agreements have been achieved regarding the homologies of the epiblast and coleorhiza, which have been debated for centuries (see reviews Brown, 1965, 1960). The homology of coleoptile is a more controversial topic. The grass coleoptile has been proposed as a part of the cotyledon, a leaf (first, second, or third leaf), a leaf sheath, or an innovation of grass that does not have a counterpart in the dicot embryo (see reviews Brown, 1965, 1960).

The coleoptile primordium was formed from the ventral side of the scutellum; the epiblast and coleorhiza, and the lateral and ventral scales that are covering the coleoptile, are directly derived from the scutellum, and there was no primordium differentiated for forming these structures (Xu et al., 1999; Itoh et al., 2005; Brown, 1960, 1965). Mutants that showed specific embryo defects, such as osnf-yb7, may provide new insights for the debating. The entire embryonic envelope development was impaired in osnf-yb7: coleoptile, coleorhiza, and epiblast were completely lost 
(Fig. 1I-T); the scutellum accumulated less storage reserves, and the palisade-shaped epithelial cells were not differentiated (Fig. S2A-D). A LL structure (with somewhat foliage identities) was developed from where the coleoptile and epiblast originated. LEC1 defects resulted in a heterochronic conversion for the Arabidopsis cotyledons. Given the parallel phenotypes of lec1 and osnf-yb7, we inferred that the structures of WT that transformed into the LL structure in osnf-yb7 are equivalent to the cotyledons in Arabidopsis (Fig. S12 A-C). For this consideration, the coleoptile and epiblast are most likely the cotyledon analogs in rice. The epiblast is an extension of the coleorhiza (Brown, 1960, 1965) and the midrib of the LL structure is extended from the main scutella vascular bundle (Fig. 1Q-T). Considering these, we propose a hypothesis that the entire embryonic envelope is the cotyledon of grass (Fig. S12 A-C). The expression profiles of the laser-microdissected rice embryo tissues favor the idea that the embryonic envelope is a continuum sharing similar gene expression. By reanalyzing the data generated by Sato et al. (2016), we found that mRNA profiles of the scutellum and epiblast/coleorhiza were similar but distinct from those of the shoot and root (Fig. S13).

\section{The role of the LEC1-type genes in plant chlorophyll biogenesis}

Plants, such as Arabidopsis bear chlorophyllous embryos. Chl can be detected as early as the globular stage in Arabidopsis, and the embryo turns green toward the end of the late-heart stage (Parcy et al., 1997). However, mature embryos are de-greened because Chl degradation couples with seed maturation (Smolikova et al., 2017). Previous studies showed that the mature embryos of lec1 were paler than the WT (Meinke, 1992; West et al., 1994), indicating that LEC1 acts as a positive regulator for $\mathrm{Chl}$ biogenesis. In agreement, comprehensive transcriptome analyses showed that LEC1 could transcriptionally activate dozens of chloroplast biogenesis and photosynthesis-related genes in Arabidopsis and soybean embryos (Pelletier et al., 2017; Jo et al., 2020). Like many other grass species, the embryos of rice are achlorophyllous. This study found that, in contrast to Arabidopsis, null mutation of OsNF-YB7 activated photosynthesis and Chl biosynthesis in rice. Consequently, green embryos with significant quantities of Chl were produced by the osnf-yb7 mutants (Figs. 5A-D and S6A and B). These findings indicated that the LEC1-type gene likely acts as a negative regulator in rice, but a positive regulator in Arabidopsis, for Chl biosynthesis. 
By surveying the literature, we noticed that Meinke (1992) reported that the cotyledons of lec1 mutant remained green unusually late in development. Although there was no significant Chl content difference when using whole seeds for quantification, Parcy et al. (1997) did observe that the tip of lec1 cotyledons accumulates more Chl. Moreover, the lec1;abi3 double mutant embryos produced a much higher $\mathrm{Chl}$ than the abi3 single mutant (Parcy et al., 1997). These findings challenge the concept that LEC1 positively regulates $\mathrm{Chl}$ biosynthesis and photosynthesis in Arabidopsis. It is worth noting that in the osnf-yb7 embryos, Chl also preferentially accumulated in the tip-end of the LL structure (Fig. S6A and B). Therefore, LEC1 may play a role in Chl biogenesis in different parts of the Arabidopsis cotyledons. Moreover, most studies on Arabidopsis emphasize LEC1's importance in embryo development at the maturation stage. Indeed, by comparing the transcriptomes of WT and lec1 seeds, the major difference in mRNA profiles is observed at maturation (Pelletier et al., 2017). However, LEC1 is activated within $24 \mathrm{~h}$ after fertilization (Lotan et al., 1998), its role in the early embryo developmental stages for Chl biogenesis is still unknown.

Photosynthesis and Chl biogenesis-related genes were enriched for the LEC1 binding targets (Pelletier et al., 2017), many of which were also differentially expressed in osnf-yb7, indicating that they are likely common downstream targets of the LEC1-type TFs in plants. Interestingly, we found that OsGLK1, OsPIL14, OsHY5L, and OsGNC were upregulated in the osnf-yb7 embryos (Fig. $\mathbf{5 E}-\mathbf{H})$. These TFs are important for chloroplast development and photomorphogenesis in rice (Nakamura et al., 2009; Li et al., 2019; Bai et al., 2019). The reporter gene driven by the OsGLK1 promoter was transcriptionally inactivated by OsNF-YB7 (Fig. 5J and K), further indicating that OsGLK1 could be a direct downstream target of OsNF-YB7. Previous studies have shown that LEC1 could interact with different TFs for function (Huang et al., 2015b; Boulard et al., 2017; Huang et al., 2015a; Hu et al., 2018). For example, LEC1 binds to TRICHOMELESS2 (TCL2) to repress the expression of genes involving trichome development during embryogenesis (Huang et al., 2015a). Here, we found that OsNF-YB7 interacted with OsGLK1 in vitro and in vivo (Fig. 6AD). OsGLK1 activated the expression of OsPORA and LHCB4, whereas OsNF-YB7 alone showed no impact on these genes (Fig. 6G-I). However, Coexpression of OsGLK1 and OsNF-YB7 in protoplasts repressed the ability of OsGLK1 to activate OSPORA and LHCB4 (Fig. 6G-I). Most of 
the OsGLK1-induced genes were also activated in osnf-yb7 (Fig. 5I). Therefore, we believe that OsNF-YB7 plays a dual role in regulating Chl biosynthesis and photosynthesis in rice embryos (Figs. S14A and B). First, it represses the downstream genes achieving by its function as a transcriptional inactivator; second, OsNF-YB7 can interact with TFs such as OsGLK1 to disturb their activation abilities for Chl biosynthesis and photosynthesis-related genes (Figs. S14A and B). Intriguingly, a previous study showed that OsNF-YB2 positively regulates chloroplast development in rice (Miyoshi et al., 2003), which suggests that the LEC1-type and non-LEC1-type NF-Y members can play a distinct role in Chl biogenesis.

\section{OsNF-YB7 and somatic embryogenesis}

LEC1 is essential for inducing somatic embryogenesis in Arabidopsis. Ectopic expression of LEC1 can induce the expression of embryo-specific genes in vegetative cells and initiate the formation of somatic embryos (Lotan et al., 1998). In contrast, the ability to form somatic embryos was significantly reduced for the lec1 mutant (Gaj et al., 2005). Notably, homozygous mutants of osnf-yb7 that regenerated from the calli were obtainable at $\mathrm{T}_{0}$ generation. This indicated that a de-differentiated totipotent cell carrying non-functional LEC1 alleles could still develop into a somatic embryo and be further regenerated into a viable and fertile plant. In this scenario, LEC1 is not indispensable for rice somatic embryogenesis. However, the LEC1 defects of the regenerated osnf-yb7 plant could not be transmitted to the offspring due to zygotic embryogenesis defects shown in this study, even using embryo rescue technology. This suggests that OsNF-YB7 plays a different role in zygotic and somatic embryogenesis, which deserves further investigation.

\section{Materials and Methods}

\section{Plant growth conditions}

The rice plants were grown in the experimental field of Yangzhou University in Yangzhou, Jiangsu Province, and in Lingshui, Hainan Province, China, with regular water and nutrient management. The spikelets were marked on the day of anthesis for sampling different aged embryos and endosperm. The maize used in this study was grown in the experimental field of Biogle GeneTech 
in Xishuangbanna, Yunnan Province, China. Because the seeds produced by osnf-yb7 were inviable, the mutants used were propagated asexually by ratooning as described previously (Cheng et al., 2020). The rice and maize seeds were germinated in a growth chamber. The chamber temperature was maintained at $28^{\circ} \mathrm{C}$ with $12 \mathrm{~h} / 12 \mathrm{~h}$ light/dark cycle.

\section{Generation of CRISPR/Cas9 mutants}

The osnf-yb7 mutant lines used in the study were previously generated in our lab (Niu et al., 2021). The higher-order mutants of OsNF-YB7 OsGLK1 and OsGLK2 mutants were generated using a previously described method for multiple gene editing (Cheng et al., 2020) in Zhonghua11 (O. sativa ssp. japonica). The maize lec1 mutant lines were generated by Weimibio Co. in the genetic background of KN5585. Approximately 300 bp genomic segments harboring each CRISPR targeting site was amplified by PCR and subjected to Sanger sequencing for genotyping to screen the mutants. The primers used for mutant generation and for genotyping are listed in Table S10.

\section{Sectioning, staining, and microscopic observation}

For semithin section preparations, different aged embryos of WT and osnf-yb7 were dissected under a dissecting microscope and were then fixed in FAA solution $(60 \%(\mathrm{v} / \mathrm{v})$ ethanol, $5 \%(\mathrm{v} / \mathrm{v})$ glacial acetic acid, and $5 \%(\mathrm{v} / \mathrm{v})$ formaldehyde) and subjected to vacuum pumping for $40 \mathrm{~min}$. After dehydration through an ethanol series and infiltrated with xylene for embedding in resin, the embedded samples were sectioned at $2.5 \mu \mathrm{m}$ thickness using a rotary microtome (Leica). Sections were then stained with $0.1 \%$ toluidine blue, coomassie brilliant blue, or $\mathrm{I}_{2}$-KI solution (80-mg KI, 10-mg I2 per ml) and photographed using an Olympus IX71 microscope.

The glumes were removed from rice seeds for free-hand sectioning, and then the caryopsis was carefully cut with a double-edged blade. For desiccated mature seeds, the caryopses were soaked in water at $4^{\circ} \mathrm{C}$ overnight before the experiment. The sections were then photographed or stained with TCC solution (Solarbio) according to the manufacture's protocol.

CLSM was performed as described previously (Cheng et al., 2020). Images of the PI-stained embryos were taken using an LSM710 (Zeiss) microscope with excitation/emission wavelengths 
For SEM analysis, the rice embryos were fixed overnight at $4^{\circ} \mathrm{C}$ in $2.5 \%$ glutaraldehyde in phosphate buffer (0.1 M, pH 7.0), washed three times in the phosphate buffer $(0.1 \mathrm{M}, \mathrm{pH} 7.0)$ for $15 \mathrm{~min}$ at each step, then, postfixed with $1 \%$ OsO4 in phosphate buffer for $2 \mathrm{~h}$ and washed three times in phosphate buffer. The samples were dehydrated through an ethanol series, then transferred to absolute ethanol. The dehydrated sample was coated with gold-palladium using an ion sputterer (EM SCD500, Leica) and imaged using a SEM (GeminiSEM 300, Zeiss).

\section{RNA extraction and real-time PCR assay}

Total RNA was isolated using the RNA-easy Isolation Reagent (Vazyme, R701-01). One microgram of total RNA was used for cDNA synthesis with the First Strand cDNA Synthesis kit (Vazyme, R123-01). Real-time RT-PCR was performed using the SYBR qPCR Master Mix (Vazyme, Q111-02) in the CFX Connect Real-Time PCR Detection platform (Bio-Rad). The experiments were performed using at least three biological replicates. The relative expression level of the tested genes was normalized to the rice Ubiquitin gene (GenBank accession AF184280) and calculated using the $2^{\Delta C t}$ method. The primers used for qRT-PCR were listed in Table S10.

\section{RNA-seq and differential expression analysis}

Total RNA was extracted from the 5- and 10 DAF embryos and 10 DAF endosperm of the WT and osnf-yb7. Three biological replicates for each sample were set. The qualified samples were submitted to BGI for library preparation and sequencing. The CLC Genomics Workbench 12.0 software was used for RNA-seq data analysis. The thresholds fold change $>2$ and Bonferroni $p<$ 0.05 were used for defining a DEG. The software MapMAN was used for MapMAN pathway analysis (Usadel et al., 2009). The online tool AgriGo 2.0 was used for GO analysis (Du et al., 2010). TBtools was used for heatmap generation (Chen et al., 2020).

\section{Hormone extraction and quantification}

First, approximately 0.1-g embryo was collected from the WT and osnf-yb7 for hormone extraction and quantification. Then, the contents of IAA, $A B A, G A_{1}$, and $G_{4}$ were measured by 
high-performance liquid chromatography-tandem mass spectrometry (Agilent, 1290) according to the previously described procedures (Cheng et al., 2020).

\section{Yeast two-hybrid assays}

The coding sequences (CDS) of OsGLK1 and OsNF-YB7 were cloned into the pGADT7 and pGBKT7, respectively. The constructs were cotransformed into yeast strain AH109 using Frozen-EZ Yeast Transformation II kit according to the manufacturer's protocol. The empty pGADT7 and pGBKT7 vectors were cotransformed in parallel as negative controls. The transformants were first selected on synthetic dropout medium (SD/-Trp-Leu) plates. Then, we tested protein-protein interactions using selective SD/-Trp-Leu-His and SD/-Trp-Leu-His-Ade dropout medium. Interactions were observed after $3 \mathrm{~d}$ of incubation at $28^{\circ} \mathrm{C}$. The primers used for generating these constructs are listed in Table S10.

\section{Split complementary LUC assays}

Split complementary LUC assays were performed as previously described (Niu et al., 2020). The CDS of OsGLK1 and OsNF-YB7 was cloned into JW771 and JW772 vectors to generate nLUC-OsGLK and cLUC-OSNF-YB7, respectively. The constructs were introduced into Agrobacterium tumefaciens strain GV3101 and then co-infiltrated into N. benthamiana leaves, and the LUC activities were analyzed after 48-h infiltration using Tanon Imaging System (5200 Multi; Tanon). The primers used for vector construction are shown in Table S10.

\section{Bimolecular fluorescence complementary assays}

The CDS of OsGLK1 and OsNF-YB7 was cloned into the PSPYNE (nYFP) and PSPYCE (cYFP). The prepared plasmids were transformed into Agrobacterium strain GV3101, and the indicated transformant pairs were infiltrated into $N$. benthamiana leaves. After $48 \mathrm{~h}$ after infiltration, the fluorescence signal of yellow fluorescent protein (YFP) was observed with confocal microscopy (Carl Zeiss, LSM 710). Images were captured at $514 \mathrm{~nm}$ laser excitation and 519-620 nm emission for YFP. The primers used for vector construction are shown in Table S10.

\section{Co-Immunoprecipitation Assays}


Co-immunoprecipitation assays were performed in the Rice protoplast system as described (Zhang et al., 2011). The CDS of OsGLK1 and OsNF-YB7 was cloned into pUC19-35S-FLAG-RBS vector and pJIT163-GFP driven by 35S promoter, respectively. Ten micrograms of plasmid DNA (OsGLK-GFP, GFP, and OsNF-YB7-Flag) was transformed or cotransformed into 200- $\mu$ l protoplasts and incubated in WI buffer for $12 \mathrm{~h}$. The protoplasts were collected and lysed in $500-\mu \mathrm{l}$ lysis buffer (0-mM Tris-HCl, 150-mM NaCl, 5-mM EDTA [pH 8.0], 1\% NP-40, 0.1-mM PMSF). The extracts were incubated with GFP-Trap agarose beads at $4^{\circ} \mathrm{C}$ for $3 \mathrm{~h}$ and washed three times with washing lysis buffer. Samples were boiled in an SDS protein-loading buffer. Immunoblots were detected by corresponding primary antibodies (anti-GFP, ABclonal no. AE012; anti-Flag, Sigma no. F3165). The primers used for vector construction are shown in Table S10.

\section{Dual-Luciferase Reporter assays}

The OsGLK1, LHCB4, and PORA promoter sequences were amplified from ZH11 genomic DNA and cloned into the pGreenll 0800-LUC vector (Hellens et al., 2005) as a reporter (pGLK1::LUC, pLHCB4::LUC, pPORA::LUC); the OsGLK1-GFP and OsNF-YB7-Flag constructs as effectors. The reporters and effectors were transfected into rice protoplasts in different combinations and incubated overnight. Firefly LUC and REN activities were measured using the Dual-Luciferase Reporter Assay Kit (Vazyme) following the manufacturer's instructions, and LUC:REN ratios were calculated and presented. The primers used for generating these constructs are listed in Table $\mathbf{S 1 0 .}$

\section{Chlorophyll measurement and confocal imaging}

One hundred micrograms of embryo of the indicated genotypes were extracted in $3 \mathrm{ml}$ of $100 \%$ dimethyl sulphoxide (DMSO) and incubated at $65^{\circ} \mathrm{C}$ for $1 \mathrm{~h}$. Then, the absorbance values at 648.2 and $664.9 \mathrm{~nm}$ wavelengths were measured by spectrophotometry, and then total chlorophyll content was calculated (Barnes et al., 1992).

Chlorophyll autofluorescence signal was detected using confocal microscopy (Carl Zeiss, LSM 710), excitation $633 \mathrm{~nm}$; emission 625-730 nm. 
We thank Prof. Hengxiu Yu and Dr. Chao Zhang for kindly providing the positive and negative control vectors for dual-luciferase reporter assays.

\section{Funding}

This research was supported by grants from the National Natural Science Foundation of China (32170344 and 31701392), the Science Fund for Distinguished Young Scholars of Jiangsu Province (BK20180047), the Six Talent Peaks Project in Jiangsu Province (NY-142) and the Priority Academic Development of Jiangsu Higher Education Institutions.

\section{Author contributions}

C.C. conceived the project. C.C., B.N., Z.Y., and Q-Q.L. designed the research. B.N., Z.Y., T.B., Z.E., Q.Y, X.X, J.Z., Z.Z. X.L, and Q.L. performed the experiments and analyzed the data. C.C., Z.Y and B.N. wrote the manuscript.

\section{Figure Legends}

Figure Legends

\section{Figure 1. Embryogenesis of the WT and osnf-yb7}

(A-H). The confocal laser scanning microscopy (CLSM) observations of early embryo development of the WT (A, C, E, G) and osnf-yb7 (B, D, F, H) at 2- (A, B), 3- (C, D), 4- (E, F) and $5 \mathrm{~d}$ after fertilization (DAF) (G, H). Scale bars $=100 \mu \mathrm{m}$. Sc, scutellum; $c$, coleoptile. (I-P). Scanning electron microscope (SEM) images of the WT (I-L) and osnf-yb7 (M-P) embryos at 5- (I, M, K, O) and $10 \operatorname{DAF}(\mathbf{J}, \mathbf{N}, \mathbf{L}, \mathbf{P})$. (K, L, O, P) are the magnified images of the coleoptile or the life-like structures showed in (I, J, $\mathbf{M}, \mathbf{N})$. Scale bars = $100 \mu \mathrm{m}$ in $(\mathbf{I}, \mathbf{J}, \mathbf{M}, \mathbf{N}) ;=20 \mu \mathrm{m}$ in $(\mathbf{K}, \mathbf{L}$, $\mathbf{O}$, P). Sc, scutellum; cp, coleoptile; su, suspensor; Is, lateral scale; vs, ventral scale; ep, epiblast; II, leaf-like structure.

$(\mathbf{Q}-\mathbf{T})$. Vertical $(\mathbf{Q}, \mathbf{S})$ and horizontal $(\mathbf{R}, \mathbf{T})$ cross-sections of the early developed embryos of the WT $(\mathbf{Q}, \mathbf{R})$ and osnf-yb7 (S, T). From left to right in $(\mathbf{Q}, \mathbf{S})$, embryos at 4, 5, 6, 7, and $10 \mathrm{DAF}$, respectively. From left to right in (R, T), embryos at 4, 5, 6, and $10 \mathrm{DAF}$, respectively. For the 4-, 5-, 6-, 7-, and 10-DAF-old embryos, scale bars $=50 \mu \mathrm{m}$; for the 10-DAF-old embryo, scale bars = $100 \mu \mathrm{m}$. Sc, scutellum; cp, coleoptile; cr, coleorhiza; Is, lateral scale; ep, epiblast; II, leaf-like structure.

Figure 2. Morphologically characterized leaf-like structure of osnf-yb7 after germination 
(A-H). Morphology of the WT coleoptile (A, B) and the osnf-yb7 leaf-like structure (C, D) at $120 \mathrm{~h}$ after germination. The boxed region in (A) and (C) is magnified in (B) and (D). Scale bars $=1 \mathrm{~mm}$. The dashed lines in $(\mathbf{A}, \mathbf{C})$ showed the sites of the sections in $(\mathbf{K}, \mathbf{L})$.

(E-J). Scanning electron microscopy images of the WT coleoptile $(\mathbf{E}, \mathbf{F})$, the WT first leaf $(\mathbf{G}, \mathbf{H})$, and the osnf-yb7 leaf-like structure (I, J). (E, G, I) show the tip parts of the tissues; (F, H, J) show the epidermal cells of the tissues. Scale bars $=20 \mu \mathrm{m}$. White arrows in $(\mathbf{G}, \mathbf{I})$ indicate hairs derived from the WT first leaf (G) and the leaf-like structure of osnf-yb7 (I); white arrowheads in $(\mathbf{H}, \mathbf{J})$ indicate trichomes.

$(\mathbf{K}, \mathbf{L})$. Horizontal cross-sections of the basal parts, which are indicated by the dashed lines in (A, C), of the WT (K) and osnf-yb7 (L) seedlings. Scale bars = 50 $\mu \mathrm{m}$. Cp, coleoptile; II, leaf-like structure; L1, the first leaf, L2, the second leaf. Black arrows indicate the vascular bundles.

\section{Figure 3. Transcriptome analysis of the embryos of WT and osnf-yb7 at 5- and 10 DAF}

(A). Venn diagrams of the differentially expressed genes (DEGs) identified from the WT and osnf-yb7 embryos at 5- and 10 DAF (upper panel), and the developmentally related DEGs (drDEGs) identified from the 5- and 10-DAF-old embryos of the WT and osnf-yb7 (lower panel). (B). Heat maps indicate distinct tissue-preferential expression patterns of the top-100 downregulated (left) and upregulated DEGs (right) identified from the 10-DAF-old osnf-yb7 embryos. The expression data of genes in caryopsis, embryo, endosperm, anther, panicle, seedling, coleoptile, shoot, and root were retrieved from Genevestigator.

(C, D). Many rice embryonic tissue-specific are differentially expressed in the osnf-yb7 embryos at 5- (C) and 10 DAF (D). The scutellum (Sc)-, epiblast (mixture with coleorhiza) (Sc/Cr)-, shoot (Sh)- and root (Rt)-preferential genes were identified by Itoh et al. (2016).

(E, F). Enrichment analysis of the differentially expressed transcription factor genes identified from 5- (E) and 10-DAF-old (F) embryos of osnf-yb7. Bubble size indicates the number of differentially expressed transcription factors of the indicated family.

(G). The heat map shows different GO terms were enriched in the up- and downregulated drDEGs identified from the WT and osnf-yb7 embryos.

$(\mathbf{H}, \mathbf{I})$. TTC staining of the 10-DAF-old WT $(\mathbf{H})$ and osnf-yb7 $(\mathbf{I})$ seeds. Scale bars $=1 \mathrm{~mm}$.

(J, K). Embryo morphology of the WT (J) and osnf-yb7 (K) at $\square 15$ DAF. Scale bars = $1 \mathrm{~mm}$.

\section{Figure 4. Loss-of-function of OsNF-YB7 weakens dormancy}

(A). Schematic illustration of the genes involving rice dormancy regulation.

(B). The heat map shows the expression of rice dormancy-related genes in the 5- and 10-DAF-old embryos of WT and osnf-yb7.

(C-F). Contents of $A B A(C), G A_{1}(D), G A_{4}(E)$, and the $A B A: G A$ ratio $(F)$ in the 10-DAF-old embryos of the WT and osnf-yb7. Values are means \pm SD of three biological repeats. ${ }^{* *}, p<0.01$ by Student's $t$-test.

$(\mathbf{G}, \mathbf{H})$. TTC staining of the mature embryos of the WT (G) and osnf-yb7 (H). Scale bars $=1 \mathrm{~mm}$. (I, J) Morphology of germinating seeds of the WT (upper) and osnf-yb7 (lower) at $48 \mathrm{~h} \mathrm{(I)} \mathrm{and} 120$ $\mathrm{h}(\mathrm{J})$ after imbibition. Scale bars $=5 \mathrm{~mm}$.

Figure 5. OsNF-YB7 negative regulates the expression of chlorophyll biogenesis-associated genes 
(A, B). Embryo morphologies of the WT and osnf-yb7 detached embryos at 8 DAF (A) and free-hand dissected embryos at maturation (B). Scale bars $=0.2 \mathrm{~mm}$.

(C, D). Chlorophyll levels in the WT and osnf-yb7 embryos at 15 DAF (C) and maturation (D).

(E). Heat map of the expression of TFs associates with chlorophyll biogenesis or chloroplast development of the WT and osnf-yb7. The color dots indicate $\log _{2}$ (RPKM mean) of the genes in three biological replicates.

(F-H). Expression of OsGLK1 (F), OsPIL14 (G) and OsGNC (H) in WT and osnf-yb7 at 5 DAF. The data are means \pm SD of three biological replicates.

(I). The Venn diagram shows overlaps of the upregulated genes in the osnf-yb7 embryos and in the OsGLK1 overexpression transgenic plants (OsGLK1-OE). The upregulated genes in OsGLK1-OE were generated by Nakamura et al. (2009).

(J, K). Dual-luciferase reporter assays in rice protoplasts showing that OsNF-YB7 represses the activity of GLKpro::LUC. The constructs of reporter and effectors were shown in (J). The data are means \pm SD of three biological replicates. ${ }^{* *}, p<0.01$, as determined by Student's $t$-test.

Figure 6. OsNF-YB7 interacts with OsGLK1 to repress the expression of OsPORA and LHCB4

(A). $\mathrm{Y} 2 \mathrm{H}$ assays to test the interactions of OsNF-YB7 with OsGLK1. The indicated combinations of constructs were cotransformed into yeast cells and grown on the nonselective medium SD/-L-T and selective medium SD/-L-T-H and SD/-L-T-H-A.

(B). A split complementary luciferase (LUC) confirmed the interaction between OsNF-YB7 and OsGLK1. Coexpression of the fusion OsGLK1 and the N-terminal half of LUC (GLK1-nLUC) and the fusion of the C-terminal half of LUC and OSNF-YB7 (CLUC-YB7) in the epidermal cells of $N$. benthamiana leaves induced LUC activities, whereas the epidermal cells coexpressed OsGLK1-nLUC and CLUC, nLUC and CLUC-OsNF-YB7, or nLUC and CLUC did not show LUC activities.

(C). BiFC assays showed interactions between OsNF-YB7 and OsGLK1 in nuclei. OsGLK1 was fused with the N-terminal of yellow fluorescent protein (GLK1-nYFP); OsNF-YB7 was fused with the Cterminal of YFP (YB7-cYFP). The recombinant proteins were transiently coexpressed in leaf epidermal cells of $N$. benthamiana. Fluorescence signals indicate that OsGLK1 interacted with OsNF-YB7 in the nuclei.

(D). Co-IP assays showing that OsNF-YB7 interacts with OsGLK1 in vivo. 355::OsNF-YB7:GFP (YB7-GFP) and 35S::OsGLK1:3xFlag (GLK-flag) were coexpressed in rice protoplasts and were immunoprecipitated with an anti-GFP antibody, and the immunoblots were probed with anti-GFP and anti-Flag antibodies. 35s::GFP (GFP) was a negative control.

(E, F). Expression of LHCB4 (E) and OsPORA (F) was activated in the embryos of osnf-yb7 at 10 DAF.

(G). Schematic diagrams of various constructs used in dual-luciferase reporter assays. LUC, firefly luciferase; REN, Renilla luciferase.

(H, I). Dual-luciferase reporter assays in rice protoplasts showing that OsNF-YB7 represses the activation of the LHCB4pro::LUC (H) and PORApro::LUC (I) reporters by OsGLK1. The LUC:REN ratio represents the LHCB4pro:LUC and PORApro:LUC activity relative to the internal control. Data are means \pm SD of three biological replicates. ${ }^{* *}, p<0.01$, as determined by Student's $t$-test. 
(J-M). Morphologies of the embryos produced by WT (J), osnf-yb7 (K), osnf-yb7;osglk2 double mutant (L), and osnf-yb7;osglk1;osglk2 triple mutant (M). Scale bars $=0.2 \mathrm{~mm}$.

\section{Figure 7. The maize OsNF-YB7 homologs play a similar role in embryo development}

(A). The neighbor-joint tree and expressed preference of the LEC1-type genes in Arabidopsis, rice, and maize.

(B). Growth retardation showed in the maize lec1 (double mutant of Zm00001d051697 and Zm00001d017898) mutant (right) when compared with the WT (left).

(C, D). Free-hand vertical sections of the mature WT (C) and maize lec1 (D) embryos. Scale bars = $1 \mathrm{~mm}$. Sc, scutellum; cp, coleoptile; el, epiblast-like structure; cr, coleorhiza; pl, plumule; ra, radicle; II, leaf-like structure.

(E, F). The germinated embryos of WT (E) and maize lec1 (F) after $3 \mathrm{~d}$ water imbibition. Scale bars $=1 \mathrm{~mm}$. Cp, coleoptile; el, epiblast-like structure; ra, radicle; II, leaf-like structure.

$(\mathbf{G}, \mathbf{H})$. Free-hand vertical sections of the germinated WT (G) and maize lec1 $\mathbf{( H )}$ embryos after 48 $\mathrm{h}$ water imbibition. Scale bars $=1 \mathrm{~mm}$. Sc, scutellum; cp, coleoptile; el, epiblast-like structure; pl, plumule; II, leaf-like structure.

(I, J). The side view (I) and vertical view (J) of the detached germinated embryos of the WT and maize lec1. Scale bars = $1 \mathrm{~mm}$. Sc, scutellum; $c p$, coleoptile; ra, radicle; II, leaf-like structure.

$(\mathbf{K}, \mathbf{L})$. Free-hand vertical sections of the detached WT (K) and maize lec1 (L) germinated embryos at $7 \mathrm{~d}$ after germination. Scale bars $=1 \mathrm{~mm}$. Sc, scutellum; $c p$, coleoptile; el, epiblast-like structure; pl, plumule; II, leaf-like structure.

Figure 8. Schematic illustration of the functional conservation between Arabidopsis LEC1 and rice OsNF-YB7 for embryo development

Both Arabidopsis lec1 and rice osnf-yb7 mutants show defects of embryogenesis, maturation, and dormancy. Notably, like that observed in the lec1 cotyledons, the embryonic envelope of osnf-yb7 developed into a leaf-like structure with somewhat true-leaf identities. Additionally, the rice osnf-yb7 develops chloroembryos, due to a chlorophyll-biogenesis activation in the embryo. However, the lec1 seed showed paler green coloration than the WT.

Supplemental Figure 1. Seed and embryo morphologies of the WT and two independent mutant lines of osnf-yb7

Supplemental Figure 2. Histological analysis of the embryos of the WT and osnf-yb7.

Supplemental Figure 3. Relative expression of rice dormancy-related genes and ABA responsive genes in the 10-DAF-old WT and osnf-yb7 embryos

Supplemental Figure 4. Relative expression of GA metabolic and responsive genes in the osnf-yb7 embryos.

Supplemental Figure 5. Expression of late embryogenesis abundant (LEA) genes in the embryos of WT and osnf-yb7

Supplemental Figure 6. Chlorophyll biosynthesis was activated in the embryos of osnf-yb7 Supplemental Figure 7. Photosynthesis related MapMAN pathways were enriched for the differential expressed genes (DEGs) identified from the osnf-yb7 embryos

Supplemental Figure 8. Generation of osnf-yb7, osglk1 and osglk2 high-order mutants Supplemental Figure 9. Generation of maize lec1 mutants 
Supplemental Figure 10. Light is required for chlorophyll biosynthesis in the chloroembryos produced by osnf-yb7

Supplemental Figure 11. The endosperm development was not largely affected in osnf-yb7 Supplemental Figure 12. Schematic illustration the homologies of the rice embryonic structures Supplemental Figure 13. Principle component analysis of the transcriptome profiles of the scutellum, epiblast/coleorhiza mixture, shoot, and root of the embryo in rice

Supplemental Figure 14. Schematic illustration the OsNF-YB7 regulated chlorophyll biosynthesis in the embryo of rice

Supplemental Table 1. Differentially expressed genes in the 5-DAF-old embryos of osnf-yb7. Supplemental Table 1. Differentially expressed genes in the 10-DAF-old embryos of osnf-yb7. Supplemetal Table 3. Differential expression of the embyonic tissue specific genes in osnf-yb7 Supplemental Table 4. Differentially expressed transcription factors in the os $f$-yb7 embryos. Supplemental Table 5. Development related differentially expressed genes identified between the 5- and 10-DAF-old WT embryos.

Supplemental Table 6. Development related differentially expressed genes identified between the 5- and 10-DAF-old osnf-yb7 embryos.

Supplemental Table 7. Expression of the ABA-metabolic and responsive genes in the WT and osnf-yb7 embryos.

Supplemental Table 8. Expression of the ABA-metabolic and responsive genes in the WT and osnf-yb7 embryos.

Supplemental Table 9. Differentially expressed genes in the 10-DAF-old emdosperm of osnf-yb7. Supplemental Table 10. Primers used for the present study

\section{References}

Armenta-Medina, A., Gillmor, C.S., Gao, P., Mora-Macias, J., Kochian, L. V., Xiang, D., and Datla, R. (2021). Developmental and genomic architecture of plant embryogenesis: from model plant to crops. Plant Commun. 2: 100136.

Bai, B., Lu, N., Li, Y., Guo, S., Yin, H., He, Y., Sun, W., Li, W., and Xie, X. (2019). OsBBX14 promotes photomorphogenesis in rice by activating OsHY5L1 expression under blue light conditions. Plant Sci. 284: 192-202.

Barnes, J.D., Balaguer, L., Manrique, E., Elvira, S., and Davison, A.W. (1992). A reappraisal of the use of DMSO for the extraction and determination of chlorophylls $a$ and $b$ in lichens and higher plants. Environ. Exp. Bot. 32: 85-100.

Boulard, C., Fatihi, A., Lepiniec, L., and Dubreucq, B. (2017). Regulation and evolution of the interaction of the seed B3 transcription factors with NF-Y subunits. Biochim. Biophys. Acta - Gene Regul. Mech. 1860: 1069-1078.

Brown, W. (1965). The Grass Embryo-A Rebutal. Phytomorphology: 275-284.

Brown, W. (1960). The morphology of the grass embryo. Phytomorphology: 215-223.

Cagliari, A., Turchetto-Zolet, A.C., Korbes, A.P., Maraschin, F. dos S., Margis, R., and Margis-Pinheiro, M. (2014). New insights on the evolution of Leafy cotyledon1 (LEC1) type genes in vascular plants. Genomics 103: 380-387. 
Chandler, J.W. (2008). Cotyledon organogenesis. J. Exp. Bot. 59: 2917-2931.

Chen, C., Chen, H., Zhang, Y., Thomas, H.R., Frank, M.H., He, Y., and Xia, R. (2020). TBtools: An Integrative Toolkit Developed for Interactive Analyses of Big Biological Data. Mol. Plant 13: 1194-1202.

Chen, W., Wang, W., Lyu, Y., Wu, Y., Huang, P., Hu, S., Wei, X., Jiao, G., Sheng, Z., Tang, S., Shao, G., and Luo, J. (2021). OsVP1 activates Sdr4 expression to control rice seed dormancy via the ABA signaling pathway. Crop J. 9: 68-78.

Cheng, X., Pan, M., E, Z., Zhou, Y., Niu, B., and Chen, C. (2020). The Maternally Expressed Polycomb Group Gene OsEMF2a Is Essential for Endosperm Cellularization and Imprinting in Rice. Plant Commun. 2: 100092.

Du, Z., Zhou, X., Ling, Y., Zhang, Z., and Su, Z. (2010). agriGO: a GO analysis toolkit for the agricultural community. Nucleic Acids Res. 38: W64-W70.

E, Z., Li, T., Zhang, H., Liu, Z., Deng, H., Sharma, S., Wei, X., Wang, L., Niu, B., and Chen, C. (2018). A group of nuclear factor $Y$ transcription factors are sub-functionalized during endosperm development in monocots. J. Exp. Bot. 69: 2495-2510.

Gaj, M.D., Zhang, S., Harada, J.J., and Lemaux, P.G. (2005). Leafy cotyledon genes are essential for induction of somatic embryogenesis of Arabidopsis. Planta 222: 977-988.

Guo, X., Hou, X., Fang, J., Wei, P., Xu, B., Chen, M., Feng, Y., and Chu, C. (2013). The rice GERMINATION DEFECTIVE 1 , encoding a $B 3$ domain transcriptional repressor, regulates seed germination and seedling development by integrating GA and carbohydrate metabolism. Plant J. 75: 403-416.

Harada, J.J. (2001). Role of arabidopsis LEAFY COTYLEDON genes in seed development. J. Plant Physiol. 158: 405-409.

He, Y. and Niu, D. (2019). LEAFY COTYLEDONs: Old genes with new roles beyond seed development. F1000Research 8: 1-7.

Hellens, R.P., Allan, A.C., Friel, E.N., Bolitho, K., Grafton, K., Templeton, M.D., Karunairetnam, S., Gleave, A.P., and Laing, W.A. (2005). Transient expression vectors for functional genomics, quantification of promoter activity and RNA silencing in plants. Plant Methods 1: 1-14.

Hibara, K. ichiro, Obara, M., Hayashida, E., Abe, M., Ishimaru, T., Satoh, H., Itoh, J. ichi, and Nagato, Y. (2009). The ADAXIALIZED LEAF1 gene functions in leaf and embryonic pattern formation in rice. Dev. Biol. 334: 345-354.

Hong, S.-K., Aoki, T., Kitano, H., Satoh, H., and Nagato, Y. (1995). Phenotypic diversity of 188 rice embryo mutants. Dev. Genet. $16: 298-310$.

Hu, Y., Zhou, L., Huang, M., He, X., Yang, Y., Liu, X., Li, Y., and Hou, X. (2018). Gibberellins play an essential role in late embryogenesis of Arabidopsis. Nat. Plants 4: 289298.

Huang, M., Hu, Y., Liu, X., Li, Y., and Hou, X. (2015a). Arabidopsis LEAFY COTYLEDON1 
controls cell fate determination during post-embryonic development. Front. Plant Sci. 6 : 955.

Huang, M., Hu, Y., Liu, X., Li, Y., and Hou, X. (2015b). Arabidopsis LEAFY COTYLEDON1 mediates postembryonic development via interacting with PHYTOCHROME-INTERACTING FACTOR4. Plant Cell 27: 3099-3111.

Huang, X., Peng, X., and Sun, M.X. (2017). OsGCD1 is essential for rice fertility and required for embryo dorsal-ventral pattern formation and endosperm development. New Phytol. 215 : 1039-1058.

Ishimoto, K. et al. (2019). Specification of basal region identity after asymmetric zygotic division requires mitogen-activated protein kinase 6 in rice. Development 146: dev176305.

Ito, Y., Thirumurugan, T., Serizawa, A., Hiratsu, K., Ohme-Takagi, M., and Kurata, N. (2011). Aberrant vegetative and reproductive development by overexpression and lethality by silencing of OsHAP3E in rice. Plant Sci. 181: 105-110.

Itoh, J.-I., Sato, Y., Sato, Y., Hibara, K.-I., Shimizu-Sato, S., Kobayashi, H., Takehisa, H., Sanguinet, K.A., Namiki, N., and Nagamura, Y. (2016). Genome-wide analysis of spatio-temporal gene expression patterns during early embryogenesis in rice. Development 143: 1217-1227.

Itoh, J., Nonomura, K., Ikeda, K., Yamaki, S., Inukai, Y., Yamagishi, H., Kitano, H., and Nagato, Y. (2005). Rice Plant Development: from Zygote to Spikelet. Plant Cell Physiol. 46: 23-47.

Jarvis, P. and López-Juez, E. (2013). Biogenesis and homeostasis of chloroplasts and other plastids. Nat. Rev. Mol. Cell Biol. 14: 787-802.

Jo, L., Pelletier, J.M., and Harada, J.J. (2019). Central role of the LEAFY COTYLEDON1 transcription factor in seed development. J. Integr. Plant Biol. 61: 564-580.

Jo, L., Pelletier, J.M., Hsu, S., Baden, R., Goldberg, R.B., and Harada, J.J. (2020). Combinatorial interactions of the LEC1 transcription factor specify diverse developmental programs during soybean seed development. Proc. Natl. Acad. Sci. 117: 1223-1232.

Lee, H., Fischer, R.L., Goldberg, R.B., and Harada, J.J. (2003). Arabidopsis LEAFY COTYLEDON1 represents a functionally specialized subunit of the CCAAT binding transcription factor. Proc. Natl. Acad. Sci. U. S. A. 100: 2152-6.

Lepiniec, L., Devic, M., Roscoe, T.J., Bouyer, D., Zhou, D.X., Boulard, C., Baud, S., and Dubreucq, B. (2018). Molecular and epigenetic regulations and functions of the LAFL transcriptional regulators that control seed development. Plant Reprod. 31: 291-307.

Li, Z., Mo, W., Jia, L., Xu, Y.C., Tang, W., Yang, W., Guo, Y.L., and Lin, R. (2019). Rice FLUORESCENT1 Is Involved in the Regulation of Chlorophyll. Plant Cell Physiol. 60: 23072318.

Lotan, T., Ohto, M., Yee, K.M., West, M.A.L., Lo, R., Kwong, R.W., Yamagishi, K., Fischer, R.L., Goldberg, R.B., and Harada, J.J. (1998). Arabidopsis LEAFY 

COTYLEDON1 is sufficient to induce embryo development in vegetative cells. Cell 93: 11951205.

Meinke, D.W. (1992). A Homoeotic Mutant of Arabidopsis thaliana with Leafy Cotyledons. Science 258: 1647-50.

Meinke, D.W., Franzmann, L.H., Nickle, T.C., and Yeung, E.C. (1994). Leafy cotyledon mutants of Arabidopsis. Plant Cell 6: 1049-1064.

Miyoshi, K., Ito, Y., Serizawa, A., and Kurata, N. (2003). OsHAP3 genes regulate chloroplast biogenesis in rice. Plant J. 36: 532-540.

Morita, A., Umemura, T.A., Kuroyanagi, M., Futsuhara, Y., Perata, P., and Yamaguchi, J. (1998). Functional dissection of a sugar-repressed $\alpha$-amylase gene (RAmy1A) promoter in rice embryos. FEBS Lett. 423: 81-85.

Mu, J. et al. (2008). LEAFY COTYLEDON1 Is a Key Regulator of Fatty Acid Biosynthesis in Arabidopsis. Plant Physiol. 148: 1042-1054.

Nagato, Y., Kitano, H., Kamijima, O., Kikuchi, S., and Satoh, H. (1989). Developmental mutants showing abnormal organ differentiation in rice embryos. Theor. Appl. Genet. 78: $11-15$.

Nagatoshi, Y., Mitsuda, N., Hayashi, M., Inoue, S.I., Okuma, E., Kubo, A., Murata, Y., Seo, M., Saji, H., Kinoshita, T., and Ohme-Takagi, M. (2016). GOLDEN 2-LIKE transcription factors for chloroplast development affect ozone tolerance through the regulation of stomatal movement. Proc. Natl. Acad. Sci. U. S. A. 113: 4218-4223.

Nakamura, H., Muramatsu, M., Hakata, M., Ueno, O., Nagamura, Y., Hirochika, H., Takano, M., and Ichikawa, H. (2009). Ectopic overexpression of the transcription factor osglk1 induces chloroplast development in non-green rice cells. Plant Cell Physiol. 50: 19331949.

Niu, B., Deng, H., Li, T., Sharma, S., Yun, Q., Li, Q., E, Z., and Chen, C. (2020). OsbZIP76 interacts with OsNF-YBs and regulates endosperm cellularization in rice ( Oryza sativa ). J. Integr. Plant Biol. 62: 1983-1996.

Niu, B., Zhang, Z., Zhang, J., Zhou, Y., and Chen, C. (2021). The rice LEC1-like transcription factor OsNF-YB9 interacts with SPK, an endosperm-specific sucrose synthase protein kinase, and functions in seed development. Plant J.: 1233-1246.

Nosaka, M., Nagasaki, H., Mukouhata, M., Hibara, K. -i., Sato, Y., Ashikari, M., Nagato, Y., Hayashi, K., Itoh, J. -i., Satoh-Nagasawa, N., Kitano, H., and Matsuoka, M. (2007). The small interfering RNA production pathway is required for shoot meristem initiation in rice. Proc. Natl. Acad. Sci. 104: 14867-14871.

Parcy, F., Valon, C., Kohara, A., Miséra, S., and Giraudat, J. (1997). The ABSCISIC ACID-INSENSITIVE3, FUSCA3, and LEAFY COTYLEDON1 loci act in concert to control multiple aspects of arabidopsis seed development. Plant Cell 9: 1265-1277.

Pelletier, J.M., Kwong, R.W., Park, S., Le, B.H., Baden, R., Cagliari, A., Hashimoto, 
M., Munoz, M.D., Fischer, R.L., Goldberg, R.B., and Harada, J.J. (2017). LEC1 sequentially regulates the transcription of genes involved in diverse developmental processes during seed development. Proc. Natl. Acad. Sci. 114: E6710-E6719.

Puthur, J.T., Shackira, A.M., Saradhi, P.P., and Bartels, D. (2013). Chloroembryos: A unique photosynthesis system. J. Plant Physiol. 170: 1131-1138.

Qi, D., Wen, Q., Meng, Z., Yuan, S., Guo, H., Zhao, H., and Cui, S. (2020). OsLFR is essential for early endosperm and embryo development by interacting with SWI/SNF complex members in Oryza sativa. Plant J. 104: 901-916.

Sakuraba, Y., Kim, E.Y., Han, S.H., Piao, W., An, G., Todaka, D., Yamaguchi-Shinozaki, K., and Paek, N.C. (2017). Rice Phytochrome-Interacting Factor-Like1 (OsPIL1) is involved in the promotion of chlorophyll biosynthesis through feed-forward regulatory loops. J. Exp. Bot. 68: 4103-4114.

Sato, Y., Hibara, K.-I., Nagamura, Y., Takehisa, H., Shimizu-Sato, S., Sanguinet, K.A., Itoh, J.-I., Namiki, N., and Kobayashi, H. (2016). Genome-wide analysis of spatiotemporal gene expression patterns during early embryogenesis in rice. Development 143: 1217-1227.

Satoh, N., Hong, S.K., Nishimura, A., Matsuoka, M., Kitano, H., and Nagato, Y. (1999). Initiation of shoot apical meristem in rice: Characterization of four SHOOTLESS genes. Development 126: 3629-3636.

Satoh, N., Itoh, J.I., and Nagato, Y. (2003). The SHOOTLESS2 and SHOOTLESS1 genes are involved in both initiation and maintenance of the shoot apical meristem through regulating the number of indeterminate cells. Genetics 164: 335-346.

Sazuka, T., Kamiya, N., Nishimura, T., Ohmae, K., Sato, Y., Imamura, K., Nagato, Y., Koshiba, T., Nagamura, Y., Ashikari, M., Kitano, H., and Matsuoka, M. (2009). A rice tryptophan deficient dwarf mutant, tdd1, contains a reduced level of indole acetic acid and develops abnormal flowers and organless embryos. Plant J. 60: 227-241.

Simkin, A.J., Faralli, M., Ramamoorthy, S., and Lawson, T. (2020). Photosynthesis in non-foliar tissues: implications for yield. Plant J. 101: 1001-1015.

Smolikova, G., Dolgikh, E., Vikhnina, M., Frolov, A., and Medvedev, S. (2017). Genetic and hormonal regulation of chlorophyll degradation during maturation of seeds with green embryos. Int. J. Mol. Sci. 18: 5-10.

Smolikova, G.N. and Medvedev, S.S. (2016). Photosynthesis in the seeds of chloroembryophytes. Russ. J. Plant Physiol. 63: 1-12.

Sugimoto, K., Takeuchi, Y., Ebana, K., Miyao, A., Hirochika, H., Hara, N., Ishiyama, K., Kobayashi, M., Ban, Y., Hattori, T., and Yano, M. (2010). Molecular cloning of Sdr4, a regulator involved in seed dormancy and domestication of rice. Proc. Natl. Acad. Sci. U. S. A. 107: 5792-5797.

Tao, Z., Shen, L., Gu, X., Wang, Y., Yu, H., and He, Y. (2017). Embryonic epigenetic reprogramming by a pioneer transcription factor in plants. Nature. 
Usadel, B., Poree, F., Nagel, A., Lohse, M., Czedik-Eysenberg, A., and Stitt, M. (2009). A guide to using MapMan to visualize and compare Omics data in plants: A case study in the crop species, Maize. Plant, Cell Environ. 32: 1211-1229.

Wang, J., Deng, Q., Li, Y., Yu, Y., Liu, X., Han, Y., Luo, X., Wu, X., Ju, L., Sun, J., Liu, A., and Fang, J. (2020). Transcription Factors Rc and OsVP 1 Coordinately Regulate Preharvest Sprouting Tolerance in Red Pericarp Rice. J. Agric. Food Chem. 68: 14748-14757.

Wang, P., Fouracre, J., Kelly, S., Karki, S., Gowik, U., Aubry, S., Shaw, M.K., Westhoff, P., Slamet-Loedin, I.H., Quick, W.P., Hibberd, J.M., and Langdale, J.A. (2013). Evolution of GOLDEN2-LIKE gene function in C3 and C4 plants. Planta 237: 481495.

Waters, M.T., Wang, P., Korkaric, M., Capper, R.G., Saunders, N.J., and Langdale, J.A. (2009). GLK transcription factors coordinate expression of the photosynthetic apparatus in Arabidopsis. Plant Cell 21: 1109-1128.

West, M.A.L., Yee, K.M., Danao, J., Zimmerman, J.L., Fischer, R.L., Goldberg, R.B., and Harada, J.J. (1994). LEAFY COTYLEDON1 is an essential regulator of late embryogenesis and cotyledon identity in Arabidopsis. Plant Cell 6: 1731-1745.

Xie, X., Chen, C., Shu, J., Thapa, R.K., Kohalmi, S.E., Marsolais, F., and Zou, J. (2021). LEAFY COTYLEDON1 expression in the endosperm enables embryo maturation in Arabidopsis. Nat. Commun.

Xie, Z., Li, X., Glover, B.J., Bai, S., Rao, G.Y., Luo, J., and Yang, J. (2008). Duplication and functional diversification of HAP3 genes leading to the origin of the seed-developmental regulatory gene, LEAFY COTYLEDON1 (LEC1), in nonseed plant genomes. Mol. Biol. Evol. 25 : 1581-1592.

Xu, X. Bin, Han, H.Z., Liu, X.D., Xu, S.X., Yang, J.F., and Yeung, E. (1999). The tridimensional morphology of rice embryo development with special reference to the scutellum and the coleoptile. Acta Bot. Sin. 41: 472-478.

Yi, J., Lee, Y.-S., Lee, D.-Y., Cho, M.-H., Jeon, J.-S., and An, G. (2016). OsMPK6 plays a critical role in cell differentiation during early embryogenesis in Oryza sativa. J. Exp. Bot. 67 : $2425-2437$.

Zhang, C., Zhang, J., Tang, Y., Liu, K., Liu, Y., Tang, J., Zhang, T., and Yu, H. (2021). DEEP GREEN PANICLE1 suppresses GOLDEN2-LIKE activity to reduce chlorophyll synthesis in rice glumes. Plant Physiol. 185: 469-477.

Zhang, J.-J. and Xue, H.-W. (2013). OsLEC1/OsHAP3E participates in the determination of meristem identity in both vegetative and reproductive developments of rice. J. Integr. Plant Biol. 55: 232-49.

Zhang, Y., Su, J., Duan, S., Ao, Y., Dai, J., Liu, J., Wang, P., Li, Y., Liu, B., Feng, D., Wang, J., and Wang, H. (2011). A highly efficient rice green tissue protoplast system for transient gene expression and studying light/chloroplast-related processes. Plant Methods 7: 1-14. 

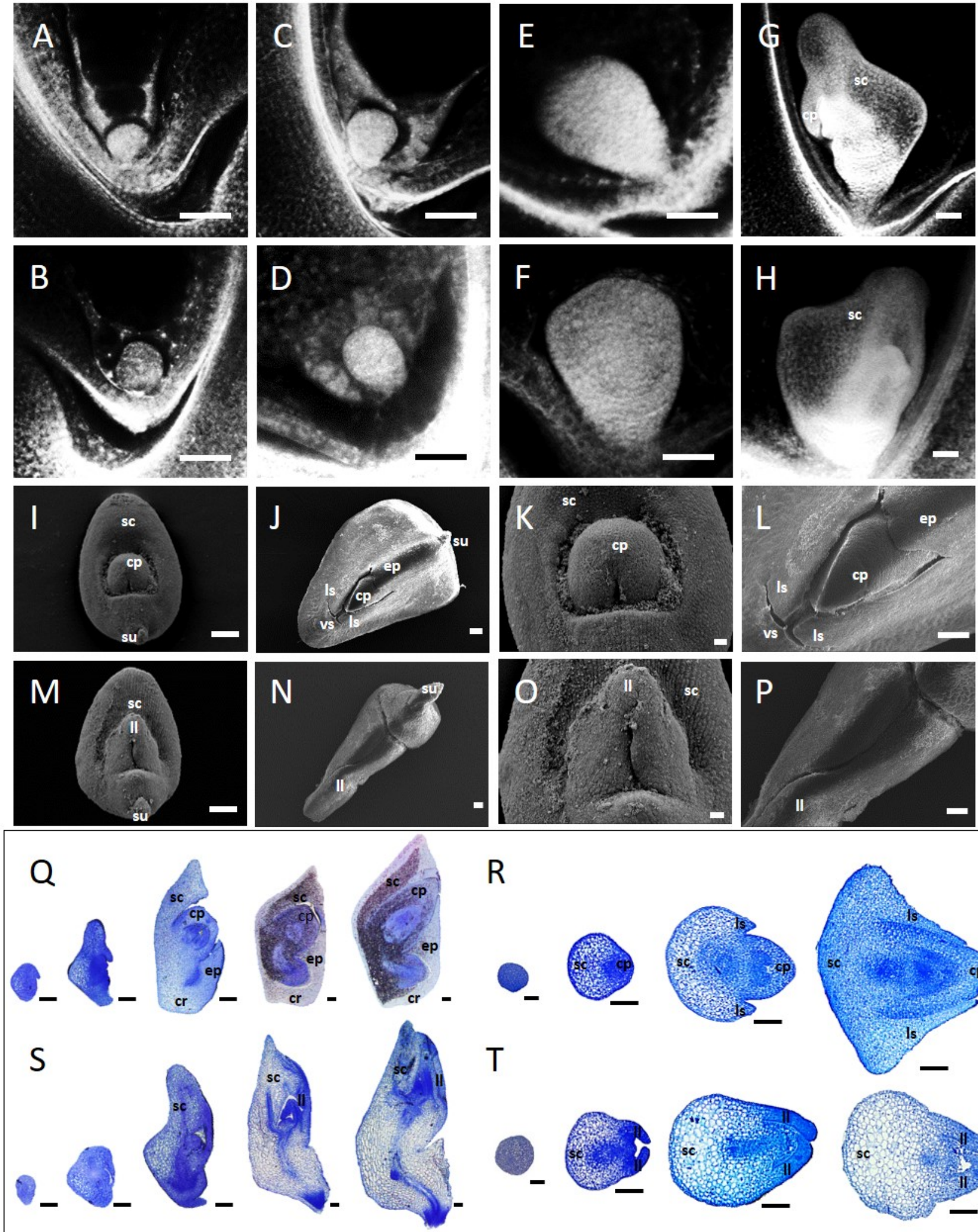

$\mathrm{T}$
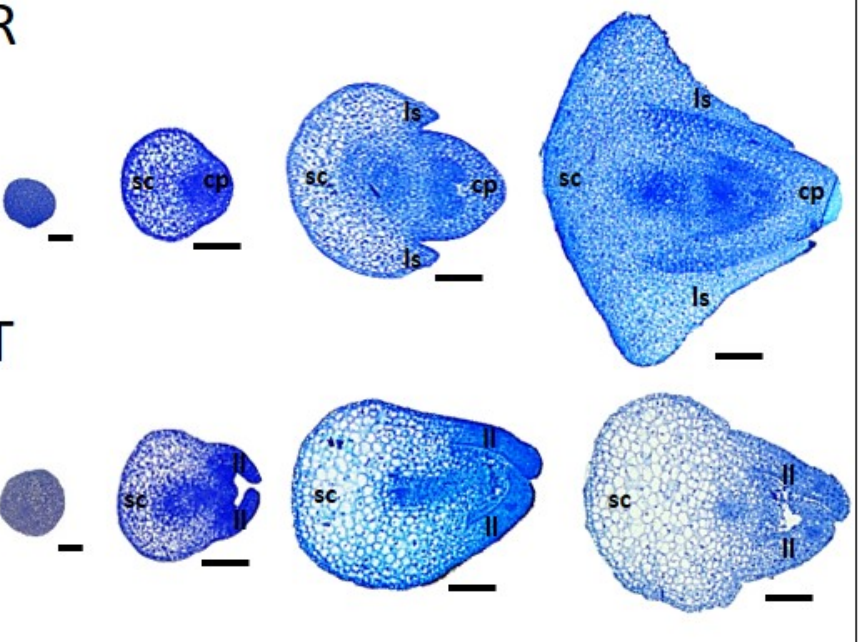

Figure 1. Embryogenesis of the WT and osnf-yb7

(A-H). The confocal laser scanning microscopy (CLSM) observations of early embryo development of the WT (A, C, E, G) and osnf-yb7 (B, D, F, H) at 2- (A, B), 3- (C, D), 4- (E, F) and $5 \mathrm{~d}$ after fertilization (DAF) (G, H). Scale bars = $100 \mu \mathrm{m}$. Sc, scutellum; cp, coleoptile.

(I-P). Scanning electron microscope (SEM) images of the WT (I-L) and osnf-yb7 (M-P) embryos at 5- (I, M, K, O) and $10 \operatorname{DAF}(\mathrm{J}, \mathrm{N}, \mathrm{L}, \mathrm{P})$. (K, L, O, P) are the magnified images of the coleoptile or the life-like structures showed in (I, J, M, N). Scale bars = $100 \mu \mathrm{m}$ in (I, J, M, N); = $20 \mu \mathrm{m}$ in (K, L, O, P). Sc, scutellum; cp, coleoptile; su, suspensor; Is, lateral scale; vs, ventral scale; ep, epiblast; II, leaf-like structure.

$(\mathbf{Q}-\mathbf{T})$. Vertical $(\mathbf{Q}, \mathbf{S})$ and horizontal $(\mathbf{R}, \mathbf{T})$ cross-sections of the early developed embryos of the $\mathbf{W T}(\mathbf{Q}, \mathbf{R})$ and osnf-yb7 (S, T). From left to right in $(\mathbf{Q}, \mathbf{S})$, embryos at 4, 5, 6, 7, and $10 \mathrm{DAF}$, respectively. From left to right in $(\mathbf{R}$, T), embryos at 4, 5, 6, and 10 DAF, respectively. For the 4-, 5-, 6-, 7-, and 10-DAF-old embryos, scale bars $=50$ $\mu \mathrm{m}$; for the 10-DAF-old embryo, scale bars $=100 \mu \mathrm{m}$. Sc, scutellum; $c p$, coleoptile; cr, coleorhiza; Is, lateral scale; ep, epiblast; II, leaf-like structure. 


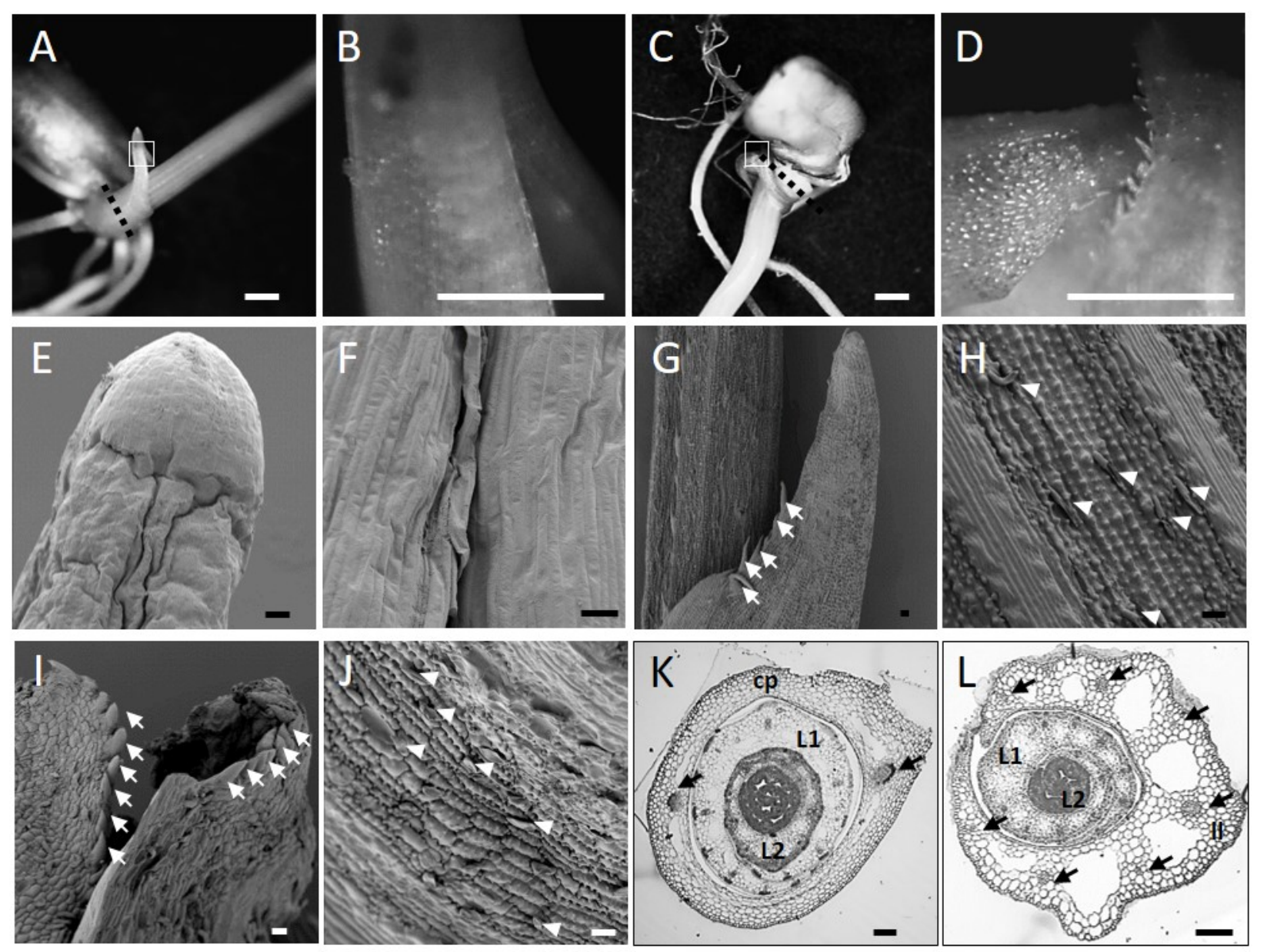

Figure 2. Morphologically characterized leaf-like structure of osnf-yb7 after germination (A-H). Morphology of the WT coleoptile (A, B) and the osnf-yb7 leaf-like structure (C, D) at $120 \mathrm{~h}$ after germination. The boxed region in (A) and (C) is magnified in (B) and (D). Scale bars $=1 \mathrm{~mm}$. The dashed lines in $(A, C)$ showed the sites of the sections in $(K, L)$.

(E-J). Scanning electron microscopy images of the WT coleoptile (E, F), the WT first leaf $(\mathrm{G}, \mathrm{H})$, and the osnf$y b 7$ leaf-like structure $(\mathbf{I}, \mathbf{J})$. $(\mathbf{E}, \mathbf{G}, \mathbf{I})$ show the tip parts of the tissues; $(\mathbf{F}, \mathbf{H}, \mathbf{J})$ show the epidermal cells of the tissues. Scale bars $=20 \mu \mathrm{m}$. White arrows in $(G, I)$ indicate hairs derived from the WT first leaf $(G)$ and the leaf-like structure of osnf-yb7 (I); white arrowheads in ( $\mathrm{H}, \mathrm{J})$ indicate trichomes.

$(\mathrm{K}, \mathrm{L})$. Horizontal cross-sections of the basal parts, which are indicated by the dashed lines in $(\mathrm{A}, \mathrm{C})$, of the WT (K) and osnf-yb7 (L) seedlings. Scale bars $=50 \mu \mathrm{m}$. Cp, coleoptile; II, leaf-like structure; L1, the first leaf, $\mathrm{L} 2$, the second leaf. Black arrows indicate the vascular bundles. 
A

WT vs osnf-yb7

5 DAF upregulated 10 DAF downregulated

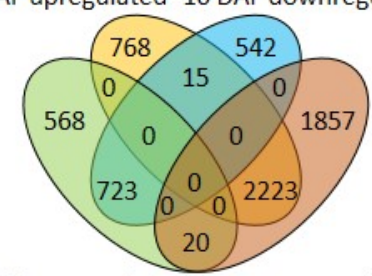

5 DAF downregulated 10 DAF upregulated

\section{DAF vs 10 DAF}

osnf-yb7 upregulated WT upregulated

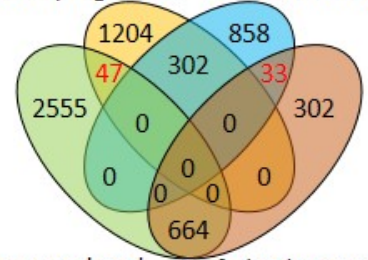

WT downregulated osnf-yb7 downregulated

D

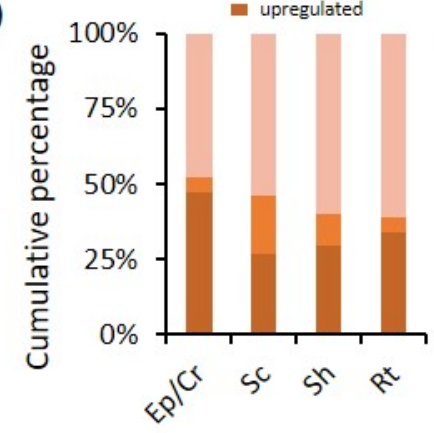

\section{E}

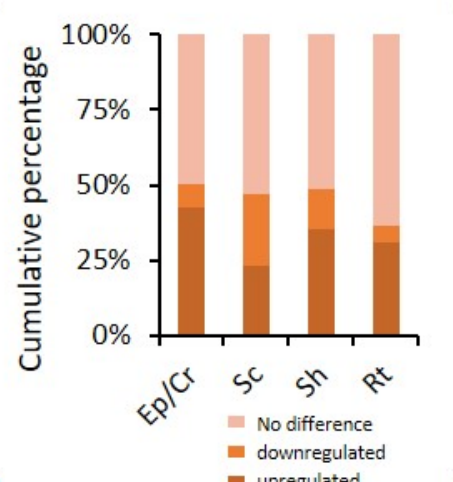

F
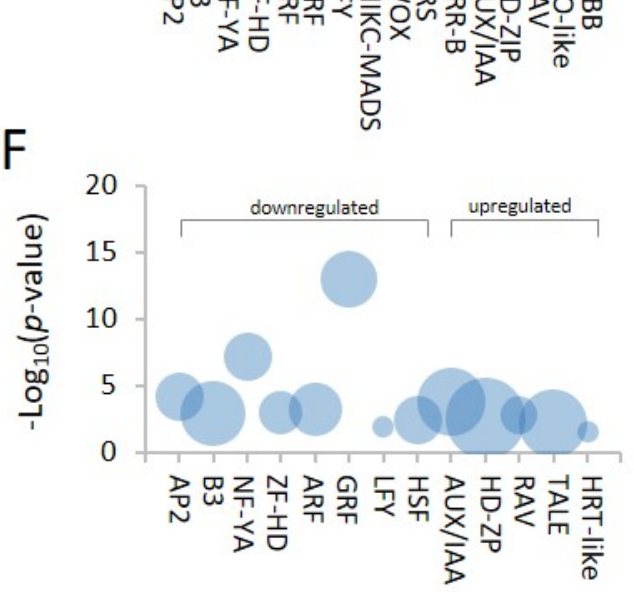

B

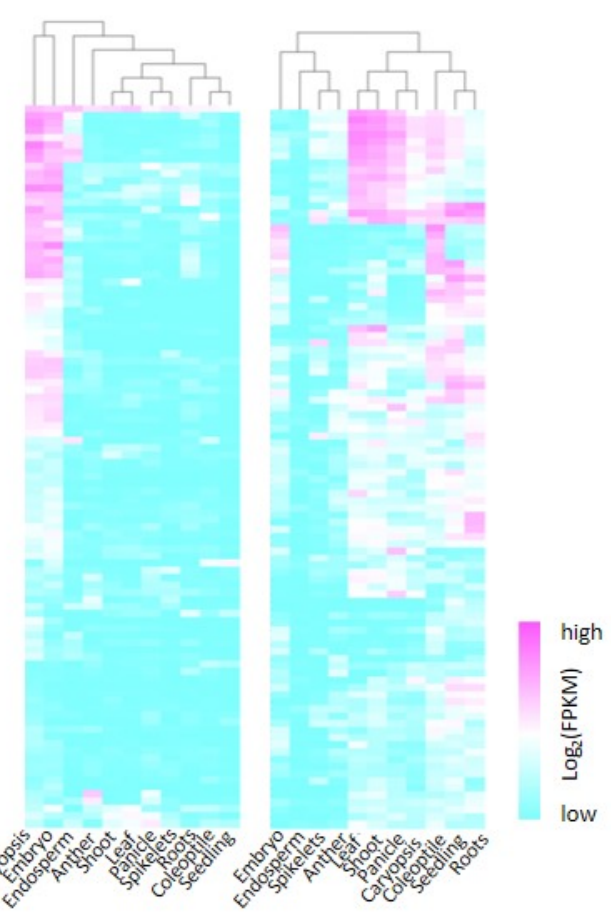

$\mathrm{H}$

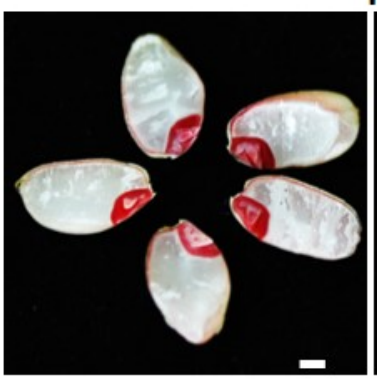

G

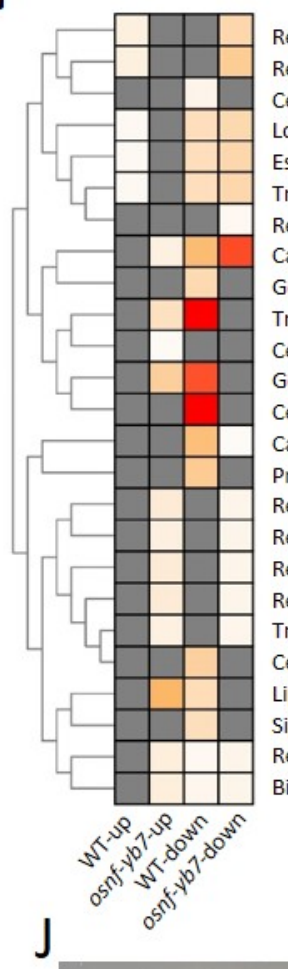

Response to stimulus Response to stress Cellular process Localization

Establishment of localization

Transport

Response to biotic stimulus

Carbohydrate metabolic process

Generation of precursor metabolites and energy

Translation

Cell cycle

Gene expression

Cellular protein metabolic process

Catabolic process

Protein metabolic process

Regulation of gene expression

Regulation of cellular process

Regulation of macromolecule metabolic proces

Regulation of metabolic process

Transcription

Cellular amino acid derivative metabolic process

Lipid metabolic process

Signal transduction

Regulation of biological process

Biological regulation

$\bigsqcup$

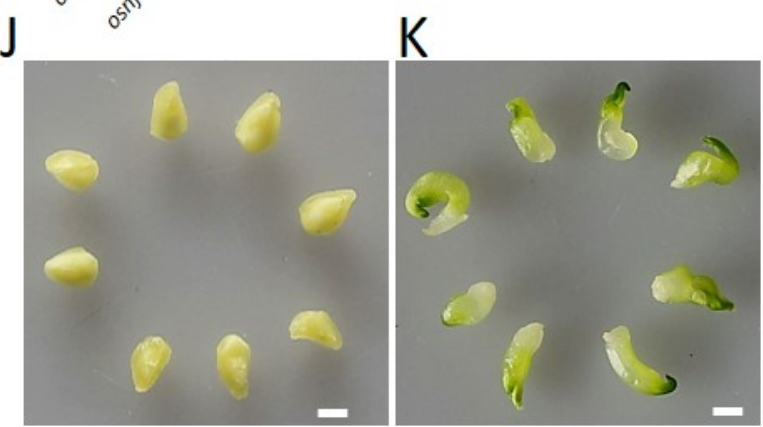

Figure 3. Transcriptome analysis of the embryos of WT and osnf-yb7 at 5- and 10 DAF

(A). Venn diagrams of the differentially expressed genes (DEGs) identified from the WT and osnf-yb7 embryos at 5- and 10 DAF (upper panel), and the developmentally related DEGs (drDEGs) identified from the 5- and 10-DAFold embryos of the WT and osnf-yb7 (lower panel).

(B). Heat maps indicate distinct tissue-preferential expression patterns of the top-100 downregulated (left) and upregulated DEGs (right) identified from the 10-DAF-old osnf-yb7 embryos. The expression data of genes in caryopsis, embryo, endosperm, anther, panicle, seedling, coleoptile, shoot, and root were retrieved from Genevestigator.

(C, D). Many rice embryonic tissue-specific are differentially expressed in the osnf-yb7 embryos at 5- (C) and 10 DAF (D). The scutellum (Sc)-, epiblast (mixture with coleorhiza) $(\mathrm{Sc} / \mathrm{Cr}$ )-, shoot (Sh)- and root (Rt)-preferential genes were identified by Itoh et al. (2016).

(E, F). Enrichment analysis of the differentially expressed transcription factor genes identified from 5- (E) and 10DAF-old (F) embryos of osnf-yb7. Bubble size indicates the number of differentially expressed transcription factors of the indicated family.

(G). The heat map shows different GO terms were enriched in the up- and downregulated drDEGs identified from the WT and osnf-yb7 embryos.

$(\mathrm{H}, \mathrm{I})$. TTC staining of the 10-DAF-old WT $(\mathrm{H})$ and osnf-yb7 (I) seeds. Scale bars $=1 \mathrm{~mm}$.

(J, K). Embryo morphology of the WT (J) and osnf-yb7 (K) at $\sim 15$ DAF. Scale bars $=1 \mathrm{~mm}$. 

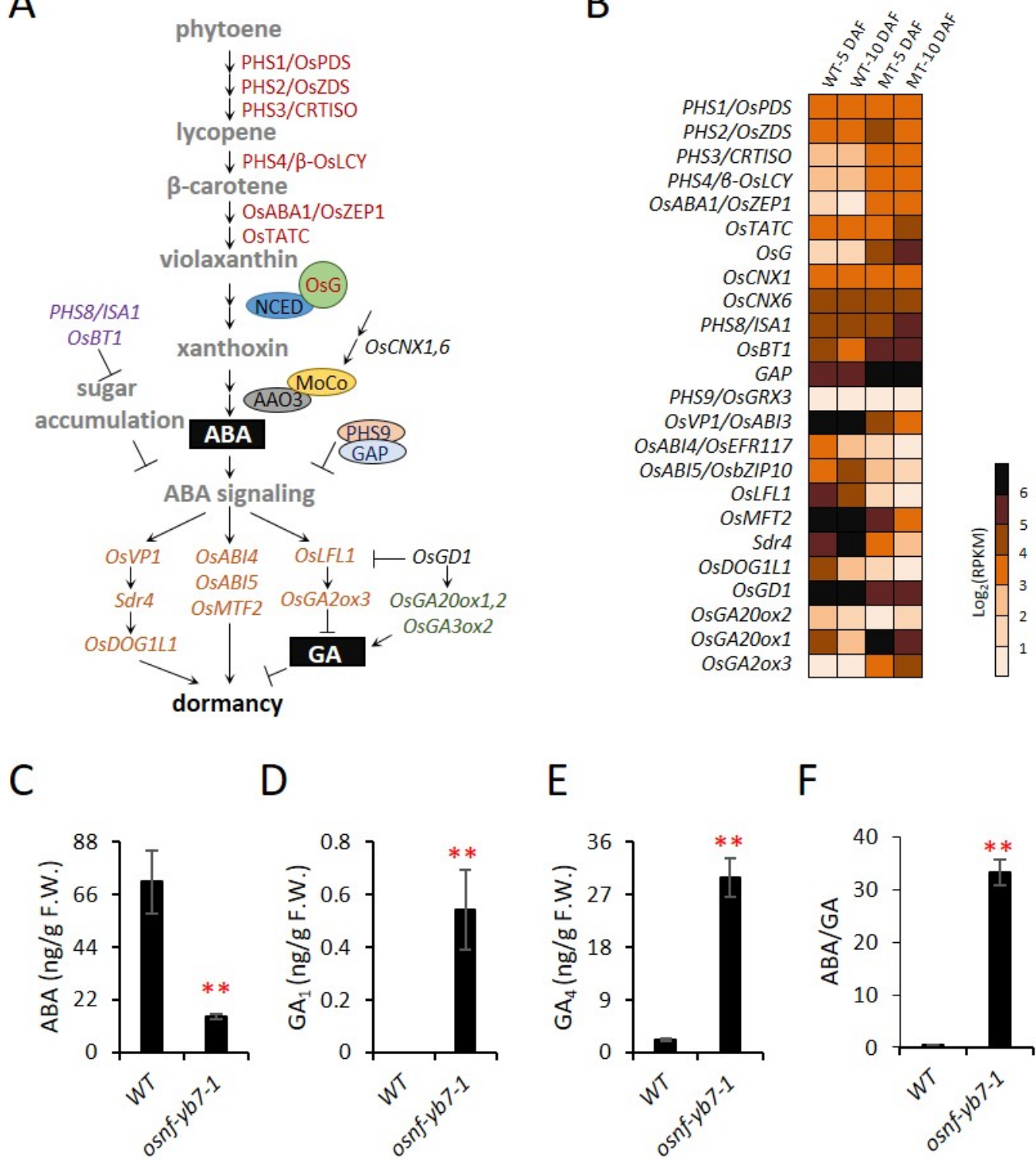

D
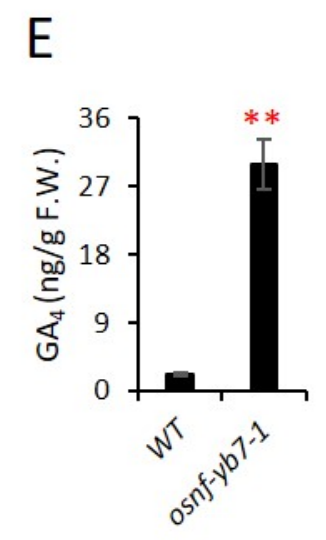

$\mathrm{F}$
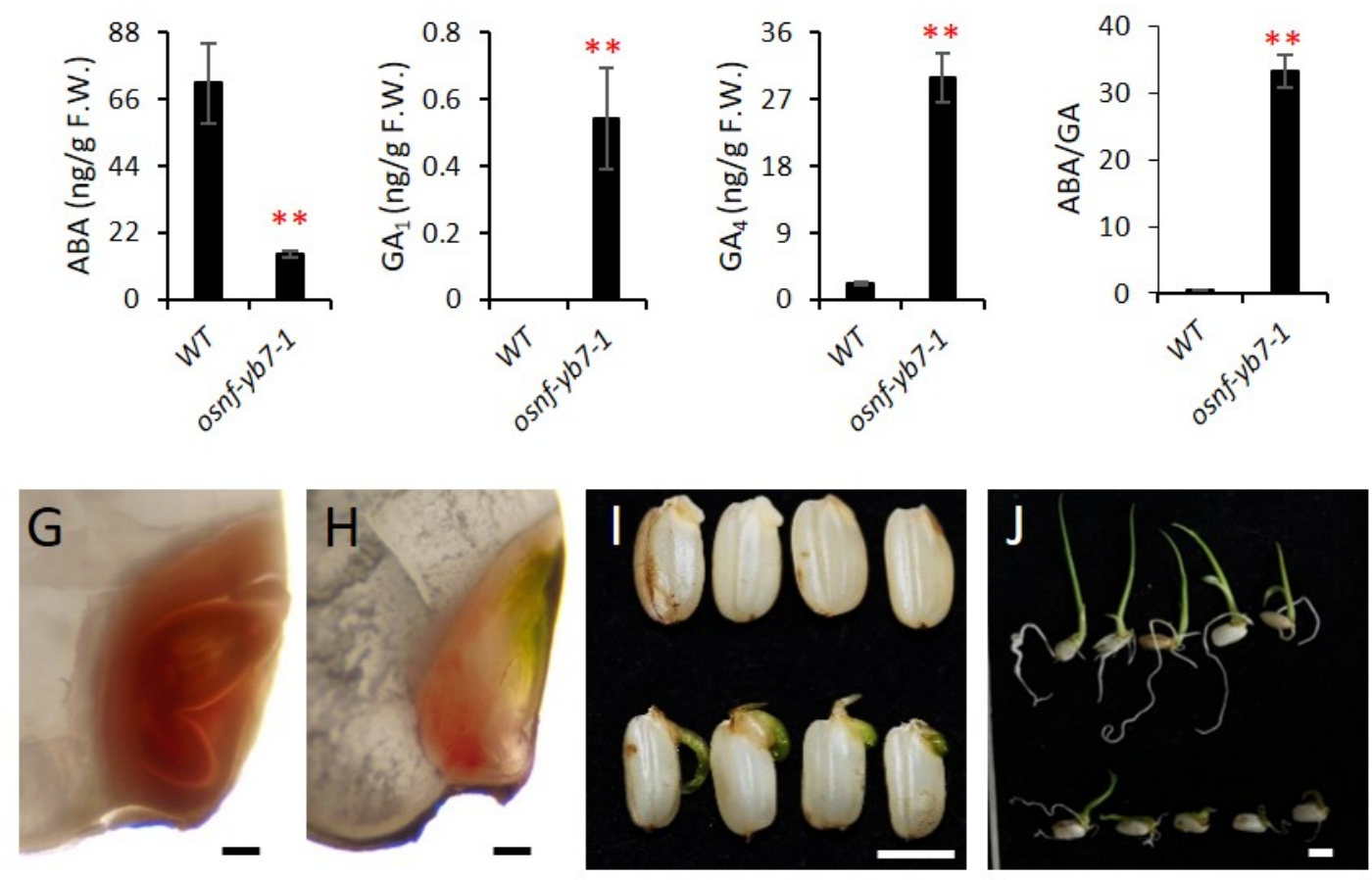

\section{Figure 4. Loss-of-function of OsNF-YB7 weakens dormancy}

(A). Schematic illustration of the genes involving rice dormancy regulation.

(B). The heat map shows the expression of rice dormancy-related genes in the 5- and 10-DAF-old embryos of WT and osnf-yb7.

(C-F). Contents of ABA (C), GA 1 (D), GA $(E)$, and the ABA:GA ratio (F) in the 10-DAF-old embryos of the WT and osnf-yb7. Values are means $\pm \mathrm{SD}$ of three biological repeats. ${ }^{* *}, p<0.01$ by Student's $t$-test.

$(G, H)$. TTC staining of the mature embryos of the WT (G) and osnf-yb7 (H). Scale bars $=1 \mathrm{~mm}$.

(I, J) Morphology of germinating seeds of the WT (upper) and osnf-yb7 (lower) at $48 \mathrm{~h}$ (I) and $120 \mathrm{~h}$ (J) after imbibition. Scale bars $=5 \mathrm{~mm}$. 
B

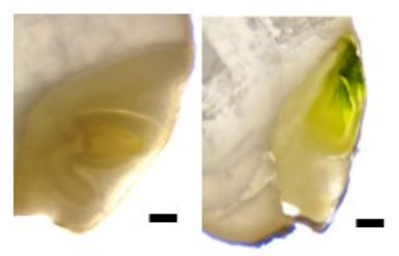

$E$

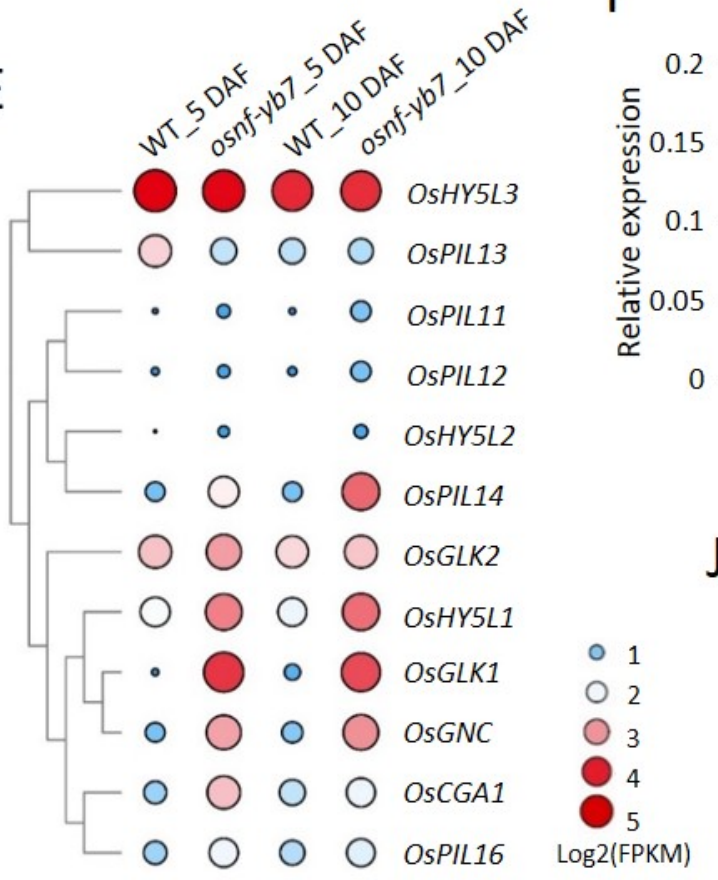

$\mathrm{F}$

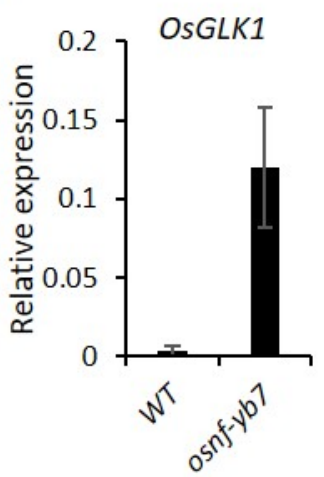

G

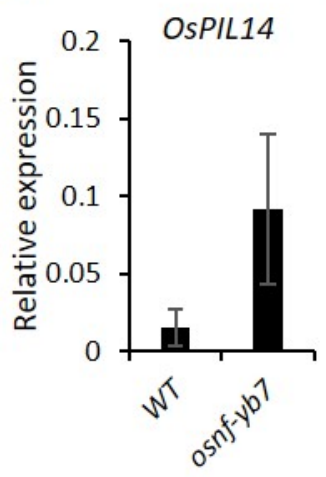

$\mathrm{H}$
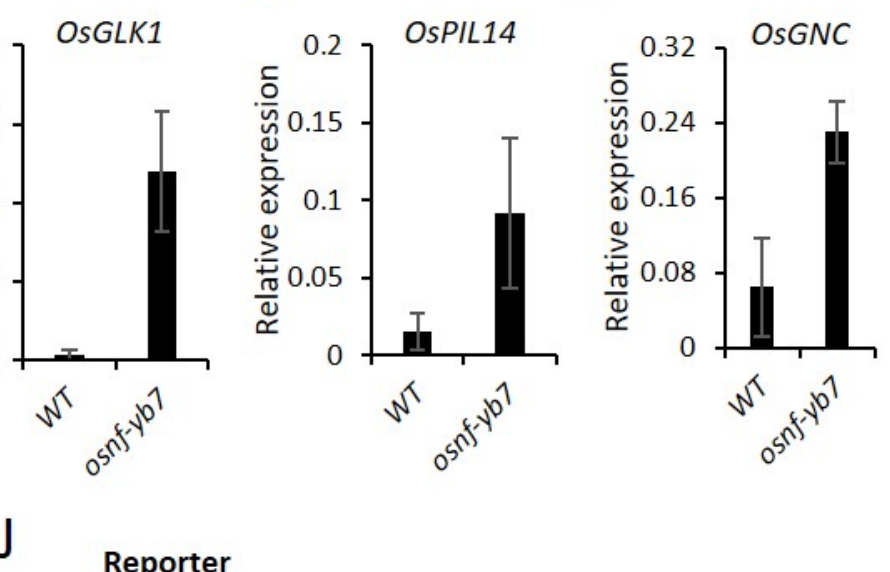

Reporter

pGLK1

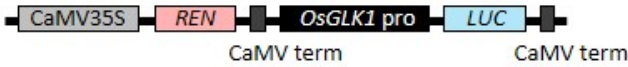

Effector

EV

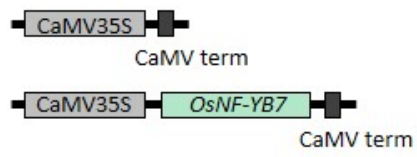

YB7

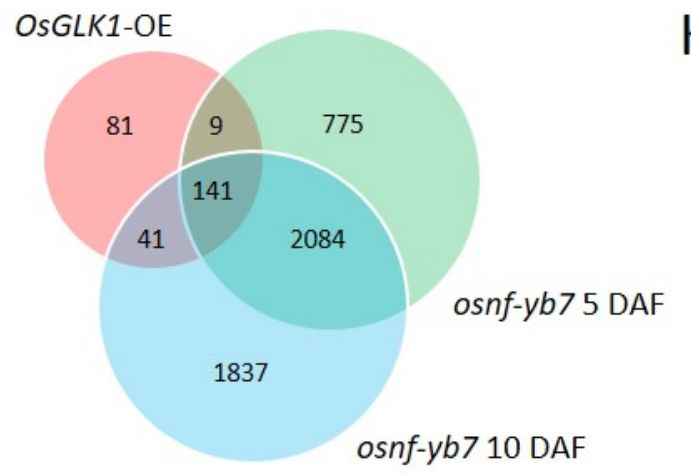

K

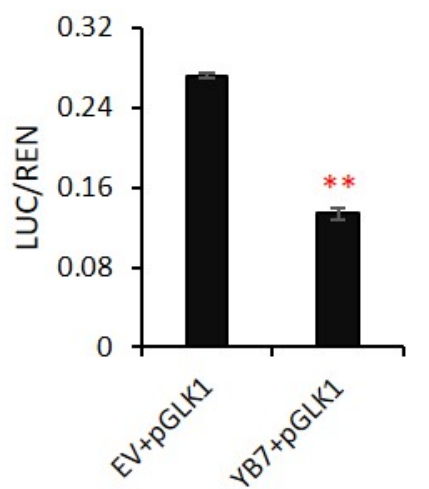

Figure 5. OsNF-YB7 negative regulates the expression of chlorophyll biogenesis-associated genes

(A, B). Embryo morphologies of the WT and osnf-yb7 detached embryos at 8 DAF (A) and free-hand dissected embryos at maturation (B). Scale bars $=0.2 \mathrm{~mm}$.

(C, D). Chlorophyll levels in the WT and osnf-yb7 embryos at 15 DAF (C) and maturation (D).

(E). Heat map of the expression of TFs associates with chlorophyll biogenesis or chloroplast development of the WT and osnf-yb7. The color dots indicate $\log _{2}$ (RPKM mean) of the genes in three biological replicates.

(F-H). Expression of OsGLK1 (F), OsPIL14 (G) and OsGNC (H) in WT and osnf-yb7 at 5 DAF. The data are means \pm SD of three biological replicates.

(I). The Venn diagram shows overlaps of the upregulated genes in the osnf-yb7 embryos and in the OsGLK1 overexpression transgenic plants (OsGLK1-OE). The upregulated genes in OsGLK1-OE were generated by Nakamura et al. (2009).

$(\mathrm{J}, \mathrm{K})$. Dual-luciferase reporter assays in rice protoplasts showing that OsNF-YB7 represses the activity of GLKpro::LUC. The constructs of reporter and effectors were shown in (J). The data are means \pm SD of three biological replicates. ${ }^{* *}, p<0.01$, as determined by Student's $t$-test. 
A

$\mathrm{AD}+\mathrm{BD}$

GLK1:AD+BD

$A D+Y B 7: B D$

GLK1:AD+YB7:BD

C
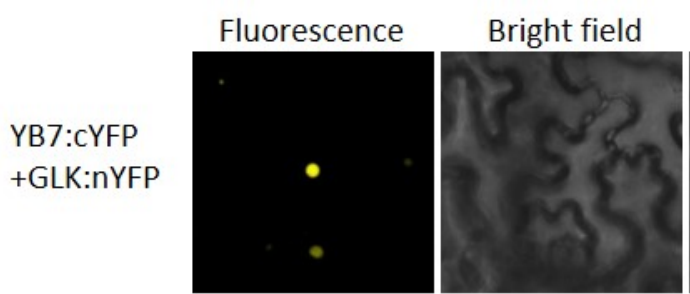

E

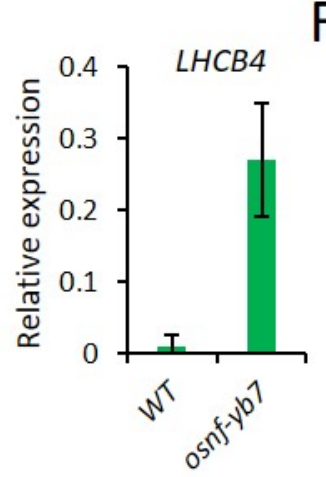

$\mathrm{F}$

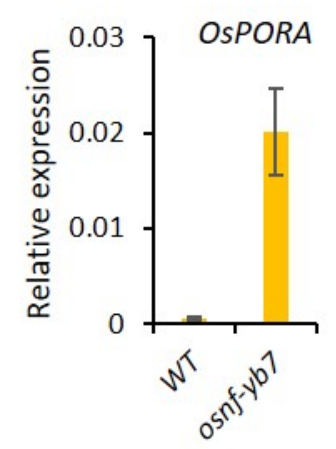

$\mathrm{H}$

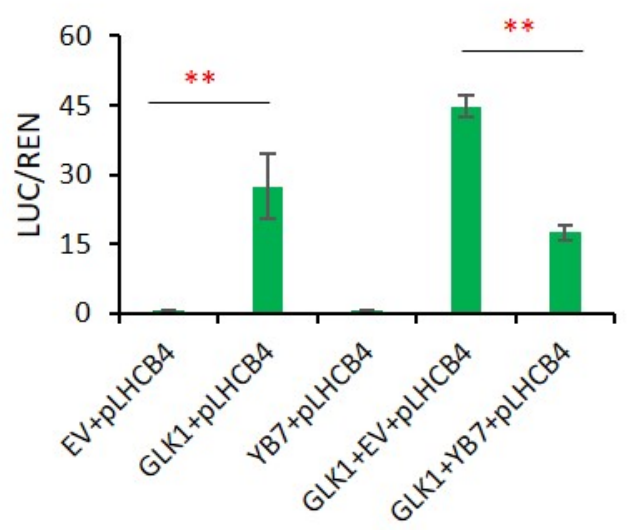

D

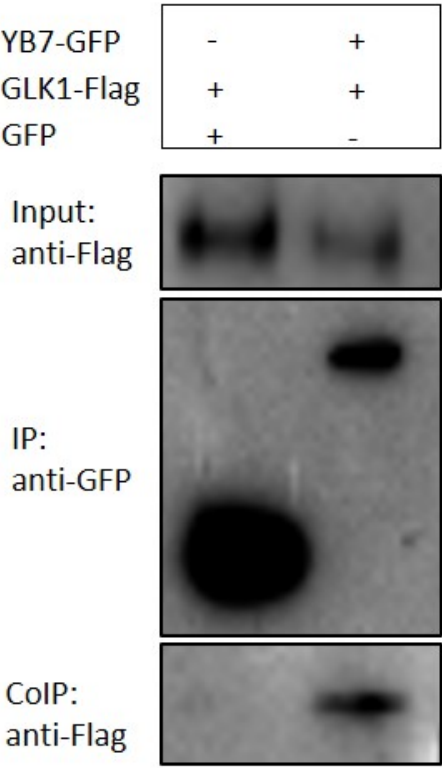

G

Reporter

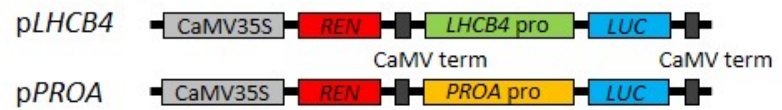

Effector

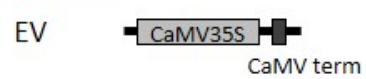

GLK1 CaMV35S OSGLK1

YB7 $=$ CaMV35S $O S N F-Y B 7-$

I

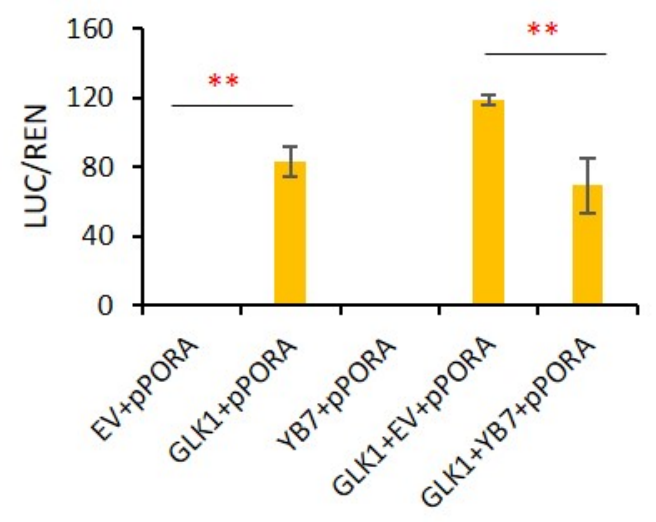

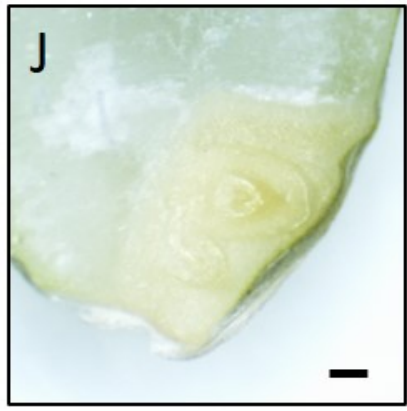

WT

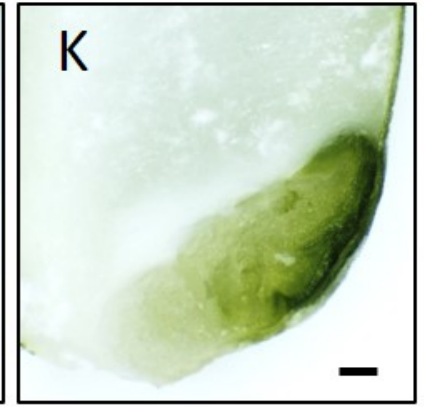

osnf-yb7

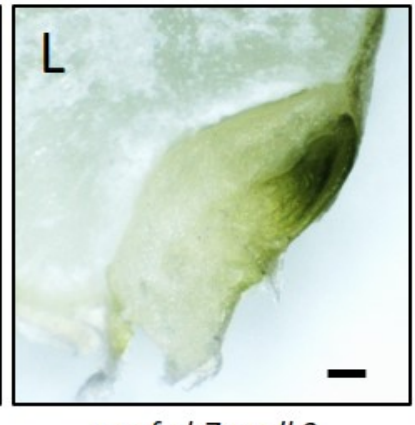

osnf-yb7 osglk2

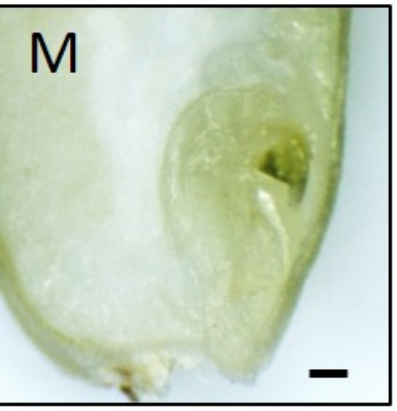

osnf-yb7 osglk1 osglk2

Figure 6. OsNF-YB7 interacts with OsGLK1 to repress the expression of OsPORA and LHCB4

(A). $\mathrm{Y} 2 \mathrm{H}$ assays to test the interactions of OsNF-YB7 with OsGLK1. The indicated combinations of constructs were cotransformed into yeast cells and grown on the nonselective medium $\mathrm{SD} /-\mathrm{L}-\mathrm{T}$ and selective medium $\mathrm{SD} /-$ L-T-H and SD/-L-T-H-A.

(B). A split complementary luciferase (LUC) confirmed the interaction between OsNF-YB7 and OsGLK1. Coexpression of the fusion OsGLK1 and the N-terminal half of LUC (GLK1-nLUC) and the fusion of the Cterminal half of LUC and OsNF-YB7 (cLUC-YB7) in the epidermal cells of $N$. benthamiana leaves induced LUC activities, whereas the epidermal cells coexpressed OsGLK1-nLUC and CLUC, nLUC and cLUC-OsNF-YB7, or nLUC and cLUC did not show LUC activities.

(C). BiFC assays showed interactions between OsNF-YB7 and OsGLK1 in nuclei. OsGLK1 was fused with the $\mathrm{N}$-terminal of yellow fluorescent protein (GLK1-nYFP); OsNF-YB7 was fused with the C-terminal of YFP (YB7cYFP). The recombinant proteins were transiently coexpressed in leaf epidermal cells of $N$. benthamiana. Fluorescence signals indicate that OsGLK1 interacted with OsNF-YB7 in the nuclei.

(D). Co-IP assays showing that OsNF-YB7 interacts with OsGLK1 in vivo. 35S::OsNF-YB7:GFP (YB7-GFP) and 35S::OsGLK1:3xFlag (GLK-flag) were coexpressed in rice protoplasts and were immunoprecipitated with an antiGFP antibody, and the immunoblots were probed with anti-GFP and anti-Flag antibodies. 35S::GFP (GFP) was a negative control.

(E, F). Expression of LHCB4 (E) and OsPORA (F) was activated in the embryos of osnf-yb7 at 10 DAF.

(G). Schematic diagrams of various constructs used in dual-luciferase reporter assays. LUC, firefly luciferase; REN, Renilla luciferase.

$(\mathrm{H}, \mathrm{I})$. Dual-luciferase reporter assays in rice protoplasts showing that OsNF-YB7 represses the activation of the LHCB4pro::LUC (H) and PORApro::LUC (I) reporters by OsGLK1. The LUC:REN ratio represents the LHCB4pro:LUC and PORApro:LUC activity relative to the internal control. Data are means \pm SD of three biological replicates. **, $p<0.01$, as determined by Student's $t$-test.

(J-M). Morphologies of the embryos produced by WT (J), osnf-yb7 (K), osnf-yb7;osglk2 double mutant (L), and osnf-yb7;osglk1;osglk2 triple mutant (M). Scale bars $=0.2 \mathrm{~mm}$. 
A

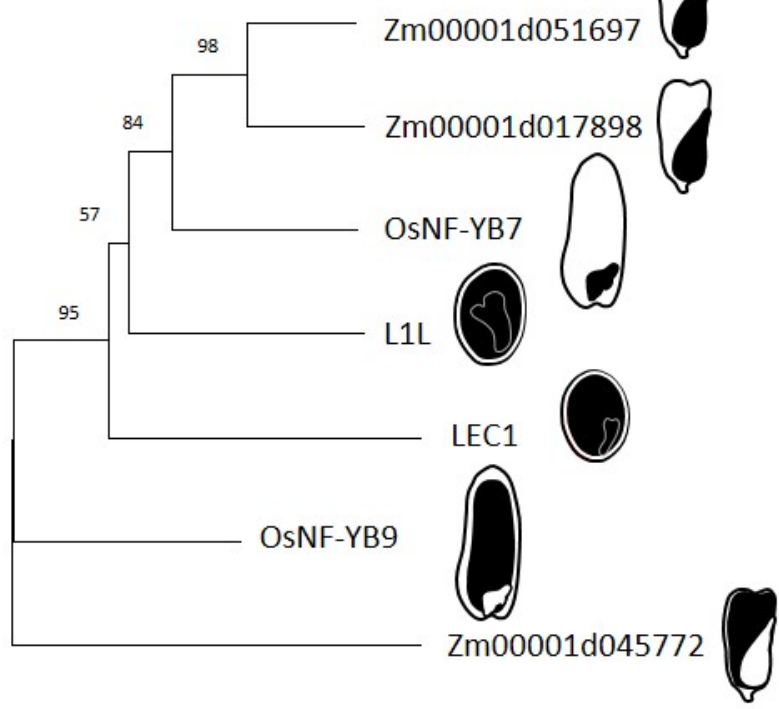

Not expressed
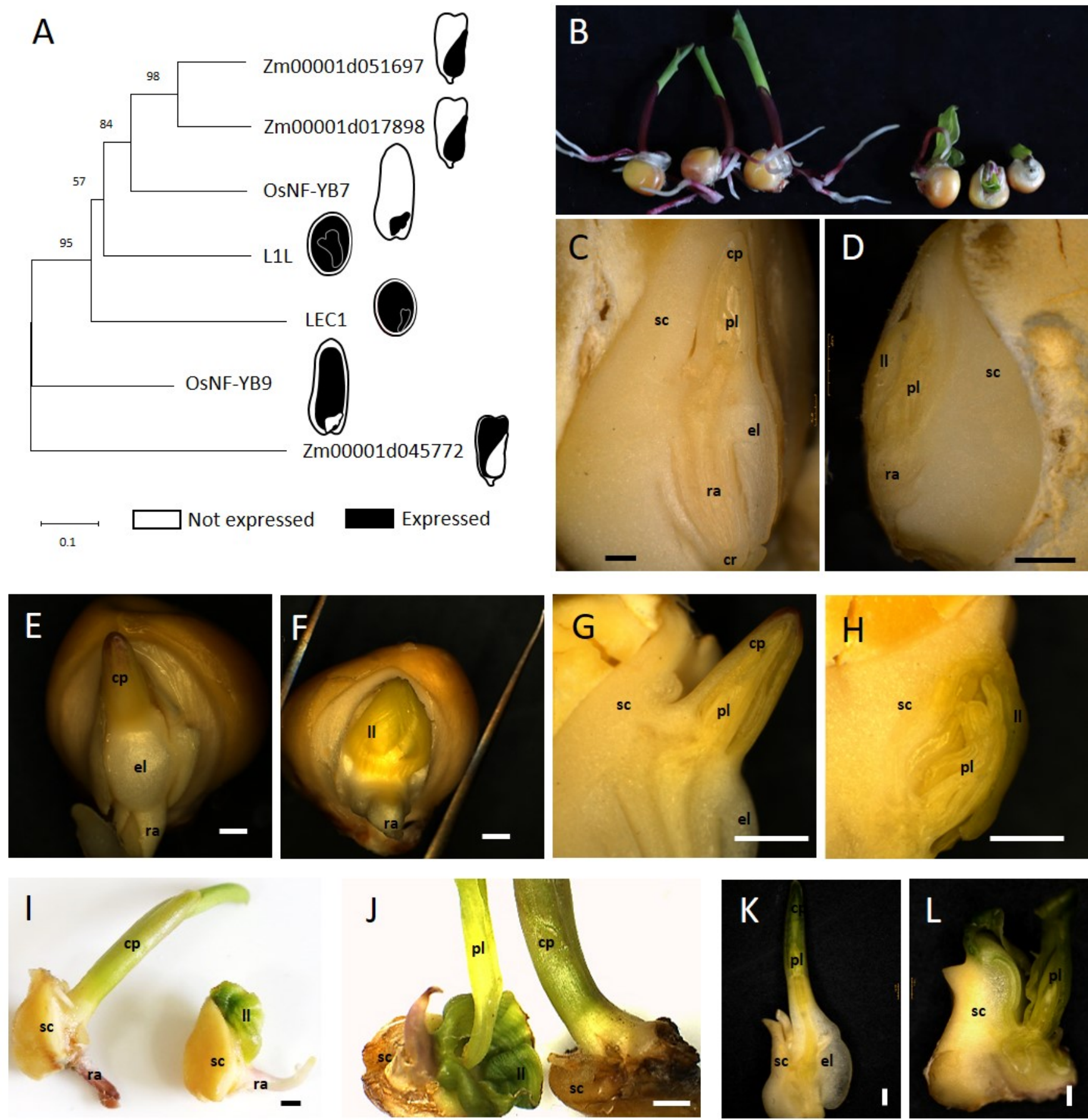

Figure 7. The maize OsNF-YB7 homologs play a similar role in embryo development

(A). The neighbor-joint tree and expressed preference of the LEC1-type genes in Arabidopsis, rice, and maize.

(B). Growth retardation showed in the maize lec1 (double mutant of Zm00001d051697 and Zm00001d017898) mutant (right) when compared with the WT (left).

(C, D). Free-hand vertical sections of the mature WT (C) and maize lec1 (D) embryos. Scale bars $=1 \mathrm{~mm}$. Sc, scutellum; cp, coleoptile; el, epiblast-like structure; cr, coleorhiza; pl, plumule; ra, radicle; Il, leaf-like structure.

$(E, F)$. The germinated embryos of WT (E) and maize lec1 (F) after $3 \mathrm{~d}$ water imbibition. Scale bars $=1$ $\mathrm{mm}$. Cp, coleoptile; el, epiblast-like structure; ra, radicle; II, leaf-like structure.

$(\mathbf{G}, \mathbf{H})$. Free-hand vertical sections of the germinated WT (G) and maize lec1 $(\mathbf{H})$ embryos after $48 \mathrm{~h}$ water imbibition. Scale bars $=1 \mathrm{~mm}$. Sc, scutellum; cp, coleoptile; el, epiblast-like structure; pl, plumule; II, leaf-like structure.

(I, J). The side view (I) and vertical view (J) of the detached germinated embryos of the WT and maize lec1. Scale bars $=1 \mathrm{~mm}$. Sc, scutellum; cp, coleoptile; ra, radicle; II, leaf-like structure.

$(\mathrm{K}, \mathrm{L})$. Free-hand vertical sections of the detached WT (K) and maize lec1 (L) germinated embryos at $7 \mathrm{~d}$ after germination. Scale bars $=1 \mathrm{~mm}$. Sc, scutellum; cp, coleoptile; el, epiblast-like structure; pl, plumule; II, leaf-like structure. 


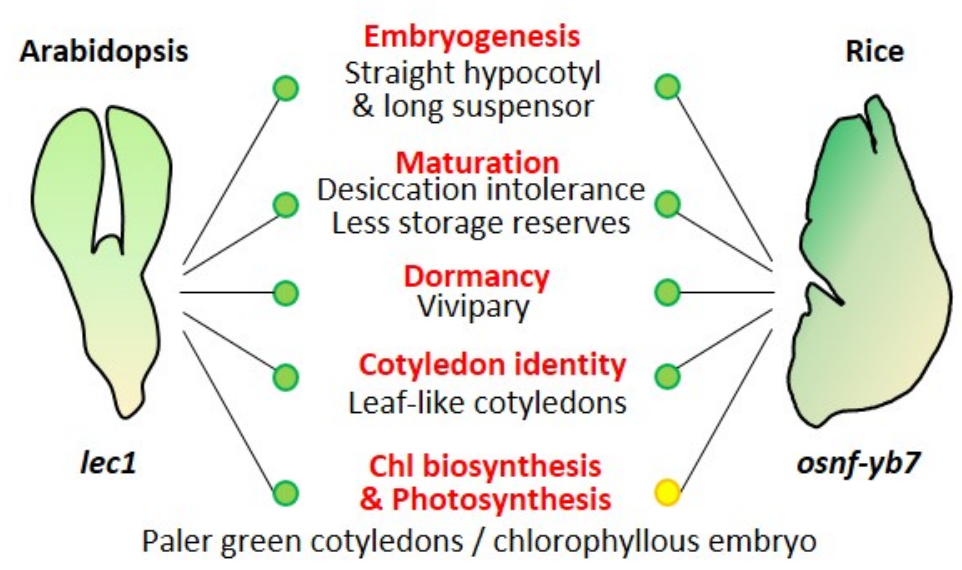

Figure 8. Schematic illustration of the functional conservation between Arabidopsis LEC1 and rice OsNF-YB7 for embryo development

Both Arabidopsis lec1 and rice osnf-yb7 mutants show defects of embryogenesis, maturation, and dormancy. Notably, like that observed in the lec1 cotyledons, the embryonic envelope of osnf-yb7 developed into a leaflike structure with somewhat true-leaf identities. Additionally, the rice osnf-yb7 develops chloroembryos, due to a chlorophyll-biogenesis activation in the embryo. However, the lec1 seed showed paler green coloration than the WT. 


\section{Supplementary Files}

This is a list of supplementary files associated with this preprint. Click to download.

- SupplementalFigures.pdf

- SupplementalTables.xlsx 